\title{
Measures on infinite dimensional vector spaces
}

\author{
By \\ Yasuo UMEMURA
}

This paper consists of three chapters. In Chap. I, using a modification of Weil's theorem on invariant measures on groups, we shall give another proof of non-existence of translationally quasiinvariant measure on infinite dimensional vector spaces, which was firstly proved by Sudakov [6]. In Chap. II, we shall prove Minlos' theorem for nuclear spaces. The original Minlos' theorem [10] required more restrictions, but actually only the nuclearity condition is necessary. In Chap. III, we shall discuss infinite dimensional Gaussian measures, and prove that we can characterize a rotationally invariant measure as a superposition of Gaussian ones. For this fact, infinite dimensionality is essential. Some applications are stated in "Introduction".

\section{Introduction}

1. In the theory of probability and the functional analysis, we are often confronted with a measure on an infinite dimensional, topological vector space (especially on a function space). In case of the product space $R^{\infty}$ of real lines $R^{1}$, a way to construct a measure was given by Kolmogorov. However, in case of a function space, we find different situations. For instance, Gaussian measures can not be constructed in anyway on the Hilbert space. Generally speaking, except on the dual space of a nuclear space, a finitely additive measure defined on the family of all finite dimensional Borel cylinders, can not always be extended to a completely additive one (Minlos' theorem).

Therefore, if we want to use measures in connection with

Doctoral thesis submitted to Kyoto University

Received July 23, 1964 
the Hilbert space, we should consider a nuclear space $L$ imbedded continuously in the Hilbert space, and construct measures on its dual space $L^{*}$.

2. On a finite dimensional vector space, the Lebesgue measure is characterized uniquely (modulo equivalence of absolute continuity with each other) as a translationally quasi-invariant one. Thus a question arises. On an infinite dimensional space also, does there exist a measure which is analogous to the Lebesgqe measure in some sense? The answer is negative. We can prove the nonexistence of measures quasi-invariant under all translations (Chap. I), while if we consider only those translations which are defined by the elements of a suitable dense subspace (for example, $L \subset L^{*}$ ), then there exist continuously many quasi-invariant measures which are not equivalent to each other.

However, under the condition that a measure should be rotationally invariant, we find that the only possible one is a superposition of Gaussian measures ( $\$ 21)$.

By this reason, it seems that Gaussian measures will play a leading role among infinite dimensional measures. Gaussian measures are also translationally quasi-invariant in the above mentioned sense. But we do not yet know whether there exists or not any translationally quasi-invariant measure which is essentially different from Gaussian ones.

3. One of the theories in which we need a measure on a function space, is the theory of stochastic processes. For instance, we consider the measure of Brownian motions (Wiener measure) on the family of all continuous functions which start from the origin, or in the differentiated form, Gaussian measure on the family of derivatives of all continuous functions. In usual, we constructed such a measure on a suitable function space case by case. But, according to the general theory, we can construct a measure $\mu$ on the dual space $L^{*}$ of a nuclear space $L$, and if necessary, we shall restrict it to a subspace of $L^{*}$, by checking the carrier of $\mu$ and the measurability of the subspace. Practically, it seems to be sufficient to discuss the case that $L=(\mathscr{Y})$ or (D) (Schwartz's function spaces), so that $L^{*}=\left(\varphi^{\prime}\right)$ or $\left(\mathscr{D}^{\prime}\right)$. 
We hope that in the near future, we shall have systematic treatments of infinite dimensional measures along this line.

4. Next in the branch of the functional analysis, using these measures we can define integrals and hence $L^{2}$-spaces over a function space. Such $L^{2}$-spaces are important for the representations of some groups.

For instance, consider the representation of the commutation relations in quantum mechanics. For a system of particles, we have the commutation relations between their momentum operators $P_{i}$ and position operators $Q_{i}$ such that;

$$
\left[Q_{i}, P_{j}\right]=i \hbar \delta_{i j}, \quad\left[Q_{i}, Q_{j}\right]=\left[P_{i}, P_{j}\right]=0 \text {. }
$$

By these relations, $P_{j}$ and $Q_{j}$ are uniquely (up to unitary equivalence) determined as the Schrödinger operators on $L^{2}\left(R^{n}\right)$;

$$
\begin{aligned}
& P_{j}: \varphi(x) \rightarrow-i \hbar \frac{\partial \varphi}{\partial x_{j}}(x) \\
& Q_{j}: \varphi(x) \rightarrow x_{j} \varphi(x) .
\end{aligned}
$$

But in case of a Boson field, we have different situations. Namely, between the operator densities which depend on a real parameter $\lambda$, the commutation relations are written symbolically as

$$
\left[Q_{\lambda}, P_{\lambda^{\prime}}\right]=i \hbar \delta\left(\lambda-\lambda^{\prime}\right), \quad\left[Q_{\lambda}, Q_{\lambda^{\prime}}\right]=\left[P_{\lambda}, P_{\lambda^{\prime}}\right]=0 ;
$$

rigorously speaking, these relations are to be formulated as

$$
\begin{aligned}
& {[Q(\varphi), P(\psi)]=i \hbar\langle\varphi, \psi\rangle} \\
& {[Q(\varphi), Q(\psi)]=[P(\varphi), P(\psi)]=0,}
\end{aligned}
$$

where $P(\varphi), Q(\varphi)$ are operators parametrized with an element of $(\varphi)$.

Then, our problem is to realize such $P(\varphi)$ and $Q(\varphi)$ as operators on the space $L^{2}\left(\varphi^{\prime}\right)$ of all square integrable functionals on $\left(\varphi^{\prime}\right)$. Though such $P(\varphi)$ and $Q(\varphi)$ can be regarded as the infinite dimensional Schrödinger operators, they are realized in continuously many ways. (Segal [1], [2] Fukutome [3]). This results from the fact that $\left(\varphi^{\prime}\right)$ has no "Lebesgue measure" but continuously many $(\mathscr{\varphi})$-quasi-invariant measures. 
In addition, an irreducible representation of $P(\varphi)$ and $Q(\varphi)$ corresponds to an $(\varphi)$-ergodic measure on $\left(\varphi^{\prime}\right)$, and hence the irreducible decomposition of a representation corresponds to the ergodic decomposition of a measure.

For another example, using the $L^{2}$-space with respect to an infinite dimensional Gaussian measure, we can represent the infinite dimensional rotation group. The author will discuss it in another paper.

5. We shall discuss the non-existence of translationally quasiinvariant measure in Chap. I,

Minlos' theorem in Chap. II, and

Gaussian measures and the characterization of rotationally invariant measures in Chap. III.

\section{Chapter I. Quasi-invariance and ergodicity}

\footnotetext{
Contents : (1) Definitions and some properties of quasi-invariance and ergodicity.

(2) Non-existence of translationally quasi-invariant Borel measure on a locally convex infinite dimensional rector space.
}

\section{§1. Quasi-invariance and ergodicity}

Let $X$ be a set, $\mathfrak{B}$ be a $\sigma$-ring which consists of subsets of $X$, $\mu$ be a measure defined on $\mathfrak{B}$, and $G$ be an onto and one-to-one transformation group on $X$. We suppose that $\mathfrak{B}$ is $G$-stable, i. e., ${ }^{\vee} g \in G,{ }^{\mathrm{V}} E \in \mathfrak{B} ; g E \in \mathfrak{B}$.

Definition 1.1. We define the transformed measure $\tau_{g} \mu$ of $\mu$ by the relation: $\tau_{g} \mu(E)=\mu(g E)$ for all $E \in \mathfrak{B}$.

\section{Definition 1.2.}

1) We say that $\mu$ is G-invariant, if $\tau_{g} \mu=\mu$ for all $g \in G$.

2) We say that $\mu$ is G-quasi-invariant, if $\tau_{g} \mu \lesssim \mu$ for all $g \in G$.

Here, $\mu_{1} \lesssim \mu_{2}$ means that $\mu_{1}$ is absolutely continuous with respect to $\mu_{2}$. If $\mu_{1} \lesssim \mu_{2}$ and $\mu_{2} \lesssim \mu_{1}$, we say that $\mu_{1}$ is equivalent with $\mu_{2}$, and denote this by $\mu_{1} \sim \mu_{2}$.

Evidently, any $G$-invariaut measure is $G$-quasi-invariant. If $\mu$ 
is equivalent with a $G$-quasi-invariant measure $\mu^{\prime}$, then $\mu$ itself is $G$-quasi-invariant. Therefore any $\sigma$-finite and $G$-invariant measure $\mu$ is equivalent with a finite and $G$-quasi-invariant measure $\mu^{\prime}$. Any linear combination with positive coefficients of $G$-quasi-inv. (or $G$ inv.) measures is $G$-quasi-inv. (or $G$-inv.), so that minimal measures are worth special interest.

Definirion 1. 3. We say that $\mu(\neq 0)$ is $G$-ergodic, if $\mu$ is minimal among G-quasi-inv. measures, i. e., the following two conditions are satisfied;

1) $\mu$ is G-quasi-inv., and

2) for any G-quasi-inv. measure $\mu^{\prime}$, the relation $\mu^{\prime} \leq \mu$ implies $\mu^{\prime}=0$ or $\mu^{\prime} \sim \mu$.

Then, a question arises: "Can any $G$-quasi-inv. measure be decomposed as a sum of $G$-ergodic measures?" This question has not been answered up to the present except some special cases, and we shall not discuss this problem in detail. (c. f. Hopf: [4], Neumann : [5])

Proposition 1.1. Let $\mu$ be a G-quasi-inv. measure, and consider the following four statements:

(1) For any G-quasi-inv. measure $\mu^{\prime}$, the relation $\mu^{\prime} \leqslant \mu$ implies $\mu^{\prime}=0$ or $\mu^{\prime} \sim \mu$.

(2) For any $\mathfrak{B}$-measurable set $E$, the relation $E=g E$ for all $g \in G$ implies that $E=\phi$ or $E=X$ (modulo $\mu$-null sets).

(3) For any $\mathfrak{B}$-measurable function $\varphi(x)$, the relation $\varphi(x)$ $=\varphi(g x)$ for all $g \in G$ implies that $\varphi(x)=$ constant for $\mu$-almost all $x$.

(4) For any $\mathfrak{B}-$ measurable set $E$, if $\mu(E)>0$, there exists a sequence $\left\{g_{n}\right\} \subset G$ such that $X=\bigcup_{k=1}^{\infty} g_{k} E$ (modulo $\mu$-nullsets).

Then, (4) is sufficient for (1), and (1) is sufficient for (2), while (2) and (3) are equivalent.

Proposition 1.1'. Let $\mu$ be a G-quasi-inv. measure, and the space $L_{\mu}^{2}(X)$ of all $\mu$-square summable functions be separable. Then the four statements of the previous proposition are equivalent with each other. 
Therefore, if $L_{\mu}^{2}(X)$ is separable, any of the four statements of Prop. 1.1 can be regarded as the definition of ergodicity. (c.f. Hopf : [4])

\section{$\S 2$. The case of vector space}

From now on, let us suppose that $X$ is a real vector space, and that $\mathfrak{B}$ satisfies the following two conditions :

a) For any $E \in \mathfrak{B}$ and $x \in X, E+x$ belongs to $\mathfrak{B}$.

b) For any $E \in \mathfrak{B}$ and real $\lambda \neq 0, \lambda E$ belongs to $\mathfrak{B}$.

However, a set which consists from one point does not always belong to $\mathfrak{B}$.

The translation $t_{y}: X \in x \rightarrow x+y \in X$ is an onto and one-to-one transformation on $X$. If $Y$ is a subspace of $X,\left\{t_{y} \mid y \in X\right\}$ forms a group, which we identify with $Y$. So, following the previous section, we can define $Y$-invariance, $Y$-quasi-invariance, and $Y$-ergodicity for measures on $X$.

Let $X^{\prime}$ be the algebraic dual space of $X$ and $R$ be a subspace of $X^{\prime}$. For any $\xi \in R$ and real $\alpha$, consider the subset of $X$;

$$
E_{\alpha, \xi}=\{x \in X \mid \xi(x)>\alpha\} \text {. }
$$

Let $\overline{\mathfrak{B}}_{R}$ be the smallest $\sigma$-ring which contains all $E_{a, \xi}$. Evidently, $\overline{\mathfrak{F}}_{R}$ satisfies the conditions a) and b) of this section.

Now, suppose that a triplet $\{X, \mathfrak{B}, \mu\}$ is given and that $\mathfrak{B} \supset \mathfrak{B}_{R}$. If we restrict $\mu$ on $\mathfrak{B}_{R}$, we get a measure $\mu_{R}\left(\equiv \mu \mid \mathfrak{B}_{R}\right)$. Though $\mu_{R}$ is a measure on $X$, it can also be regarded as a measure on the factor space $X / R^{\perp}$. Namely, let $\varphi_{R}$ be the natural homomorphism of $X$ onto $X / R^{\perp}$, and put

$$
\overline{\mathfrak{B}}_{R}=\left\{\bar{E}\left(\subset X / R^{\perp}\right) \mid \varphi_{R}^{-1}(\bar{E}) \in \mathfrak{B}_{R}\right\},
$$

then defining $\bar{\mu}_{R}$ by $\bar{\mu}_{R}(\bar{E})=\mu_{R}\left(\varphi_{R}^{-1}(\bar{E})\right)$, we can see easily that $\bar{\mu}_{R}$ is a measure on $\overline{\mathfrak{B}}_{R}$. If $\mu$ is $G$-quasi-inv., $\mu_{R}$ is also $G$-quasi-inv. Moreover under the condition: " $x-y \in R^{\perp}$ implies $g x-g y \in R^{\perp}$ for all $g \in G$ " $G$ can be regarded as a transformation gronp on $X / R^{\perp}$. Then, $G$-quasi-invariance of $\mu$ implies that of $\bar{\mu}_{R}$.

(Note: In case of the translation group $(G=Y)$, this condition is fulfilled.) 


\section{§3. A condition for absolute continuity}

In this section, we shall give a sufficient condition for absolute continuity of measures defined on $\mathfrak{B}_{R}$, supposing that $R$ is weakly separable.

Proposition 3.1. Suppose that $\left\{R_{n}\right\}$ is an increasing sequence of finite dimensional subspaces of $R$ such that $\bigcup_{n} R_{n}$ is weakly dense in $R$. Then, for any finite measure $\mu$ defined on $\mathfrak{B}_{R}$ and for $E \in \mathfrak{B}_{R}$, we have

$$
{ }^{\forall} \varepsilon>0,{ }^{3} n,{ }^{3} E_{n} \in \mathfrak{B}_{R_{n}} ; \mu\left(E \ominus E_{n}\right)<\varepsilon
$$

Here, $\ominus$ means the symmetric difference.

Proof. Let $\mathfrak{A}$ be the family of all $E$ which satisfy the condition (1). Evidently, $\mathfrak{A}$ is a $\sigma$-ring, and $E_{\alpha, \xi}=\{x \mid \xi(x)>\alpha\}$ belongs to $\mathfrak{A}$ for any $\xi \in R$ and real $\alpha$. So, $\mathfrak{A} \supset \mathfrak{B}_{R}$. (q. e.d.)

Proposition 3. $\mathbb{1}^{\prime}$. Let $L_{\mu}^{2}(X)$ be the space of all $\mu$-square summable functions defined on $X$, and $\left\{R_{n}\right\}$ be of the same meaning in Prop. 3. 1. Denoting $\mu \mid \mathfrak{B}_{R_{n}}$ by $\mu_{n}$ (instead of $\mu_{R_{n}}$ ), we can see easily that $L_{\mu_{n}}^{2}(X)$ is a closed subspace of $L_{\mu}^{2}(X)$, and that $\bigcup_{n=1}^{\infty} L_{\mu_{n}}^{2}(X)$ is dense in $L_{\mu}^{2}(X)$.

Proof. This proposition is merely another expression of Prop. 3. 1. as a statement on the $L^{2}$-space.

Corollary. Suppose that a triplet $\left\{X, \mathfrak{B}_{R}, \mu\right\}$ is given. If $R$ is weakly separable, then $L_{\mu}^{2}(X)$ is also separable, so that the four statements in Prop. 1.1 about ergodicity are equivalent with each other.

Proposition 3. 2. Suppose that $R$ is weakly sefarable, and that two finite measures $\mu$ and $\mu^{\prime}$ are given on $\mathfrak{B}_{R}$. For absolute continuity of $\mu^{\prime}$ with respect to $\mu$, it is necessary and sufficient that (using the notation of $\left\{R_{n}\right\}$ in Prop. 3.1) the following two conditions are fulfilled:

a) ${ }^{\forall} n ; \mu_{n}^{\prime} \lesssim \mu_{n}$

b) putting $\frac{d \mu_{n}^{\prime}}{d \mu_{n}}(x)=\rho_{n}(x),\left\{\sqrt{\rho_{n}(\bar{x})}\right\}$ forms a Cauchy sequence in $L_{\mu}^{2}(X)$. 
Proof of Sufficiency. Since $L_{\mu}^{2}(X)$ is complete, the Cauchy sequeince $\left\{\sqrt{\rho_{n}(x)}\right\}$ has a limit $g(x)$. Now, for any $E \in \mathfrak{B}_{R_{m}}$ and $n \geqq m$, we have

$$
\begin{aligned}
\mu^{\prime}(E) & =\int_{E} d \mu_{n}^{\prime}(x)=\int_{E} \rho_{n}(x) d \mu_{n}(x)=\int_{E} \rho_{n}(x) d \mu(x) \\
& =\left\langle C_{E} \sqrt{\rho_{n}}, C_{E} \sqrt{\rho_{n}}\right\rangle \underset{(n \rightarrow \infty)}{\longrightarrow}\left\langle C_{E} g, C_{E} g\right\rangle_{\mu}=\int_{E}|g(x)|^{2} d \mu(x) .
\end{aligned}
$$

(Here, $C_{E}(x)$ means the indicator function of the set $E$.) So, if we define $\mu^{\prime \prime}$ as $d_{\mu^{\prime \prime}}=|g(x)|^{2} d \mu$, then $\mu^{\prime}(E)=\mu^{\prime \prime}(E)$ for ${ }^{v} E \in \bigcup_{m} \mathfrak{B}_{R_{m}}$. From this, we get $\mu^{\prime}=\mu^{\prime \prime}$ on $\mathfrak{B}_{R}$ in virtue of Prop. 3.1.

Proof of Necessity. Evidently, the condition a) is necessary. Putting $\sqrt{\frac{d \mu^{\prime}}{d \mu}(x)}=g(x)$, we shall show that $\sqrt{\rho_{n}(x)} \rightarrow g(x)$ in $L_{\mu}^{2}(X)$. At first, we decompose $g(x)$ orthogonally with respect to $L_{\mu_{n}}^{2}(X)$ $\left(\equiv M_{n}\right)$ i. e..

$$
g(x)=g_{n}^{(1)}(x)+g_{n}^{(2)}(x) \quad \text { where } \quad g_{n}^{(1)} \in M_{n}, \quad g_{n}^{(2)} \in M_{n}^{\perp} .
$$

It is easy to see that $g_{n}^{(1)}(x) \geqq 0$ and $g_{n}^{(2)}(x) \geqq 0$. Since Prop. 3. $1^{\prime}$ assures $g_{n}^{(1)} \rightarrow g$ and $g_{n}^{(2)} \rightarrow 0$ respectively in $L_{\mu}^{2}(X)$, if we prove the inequality ;

$$
{ }^{\mathrm{V}} n ;\left\|\sqrt{\overline{\rho_{n}}}-g_{n}^{(1)}\right\|_{\mu} \leqq\left\|g_{n}^{(2)}\right\|_{\mu}
$$

then it is evident that $\sqrt{\rho_{n}} \rightarrow g$ in $L_{\mu}^{2}(X)$.

Proof of the Inequality (2) Let $h(x)$ be any $\mathfrak{B}_{R_{n}}$-measurable and bounded function, then

$$
\int h(x) d \mu_{n}^{\prime}(x)=\int h(x) \rho_{n}(x) d \mu(x)=\left\langle h \sqrt{\rho_{n}}, \sqrt{\rho_{n}}\right\rangle_{\mu} .
$$

Since $h(x)$ is also $\mathfrak{B}_{R}$-measurable, we have

$$
\begin{aligned}
\int h(x) d \mu_{n}^{\prime}(x) & =\int h(x) d \mu^{\prime}(x)=\int h(x)(g(x))^{2} d \mu(x) \\
& =\langle h g, g\rangle_{\mu}=\left\langle h g, g_{n}^{(1)}+g_{n}^{(2)}\right\rangle_{\mu} \\
& =\left\langle h g_{n}^{(1)}, g_{n}^{(1)}\right\rangle_{\mu}+\left\langle h g_{n}^{(2)}, g_{n}^{(2)}\right\rangle_{\mu},
\end{aligned}
$$

because $h g_{n}^{(1)} \in M_{n}$ and $g_{n}^{(2)} \in M_{n}^{\perp}$. Comparing (3) with (4), we can 
conclude that if $h(x) \geqq 0$, then $\left\langle h \sqrt{\rho_{n}}, \sqrt{\rho_{n}}\right\rangle_{\mu} \geqq\left\langle h g_{n}^{(1)}, g_{n}^{(1)}\right\rangle_{\mu}$, so that

$$
\sqrt{\rho_{n}(x)} \geqq g_{n}^{(1)}(x) \quad \text { for } \quad \mu \text {-almost all } x \text {. }
$$

Again from (3) and (4), letting $h(x) \equiv 1$, we get

$$
\int\left\{\rho_{n}(x)-\left(g_{n}^{(1)}(x)\right)^{2}\right\} d \mu(x)=\left\|g_{n}^{(2)}\right\|_{\mu}^{2}
$$

but the inequalities (5) and $g_{n}^{(1)}(x) \geqq 0$ enable us to estimate as

$$
\rho_{n}(x)-\left(g_{n}^{(1)}(x)\right)^{2}=\left(\sqrt{\rho_{n}}-g_{n}^{(1)}\right)\left(\sqrt{\rho_{n}}+g_{n}^{(1)}\right) \geqq\left(\sqrt{\overline{\rho_{n}}}-g_{n}^{(1)}\right)^{2},
$$

so that the equality (6) induces the inequality : $\left\|\sqrt{\rho_{n}}-g_{n}^{(1)}\right\|_{\mu}^{(2)}\|\leqq\| g_{n}^{(2)} \|_{\mu}^{2}$ which we have wanted to prove. (q.e.d.)

\section{$\S 4$. Uniqueness of $\mathrm{X}$-quasi-invariant measure.}

Proposition 4.1. Suppose that a $\sigma$-ring $\mathfrak{B}$ of subsets of $X$ satisfies the following condition:

$$
\begin{aligned}
& (x, y) \rightarrow x+y \quad \text { is a } \mathfrak{B} \text {-measurable mapping, } \\
& \text { i. e. for any } E \in \mathfrak{B},\{(x, y) \mid x+y \in E\} \in \mathfrak{B} \times \mathfrak{B} \text {. }
\end{aligned}
$$

Then, any two measures $\mu_{1}, \mu_{2}$ which are $\sigma$-finite and X-quasi-inv. on $\mathfrak{B}$ are equivalent with each other.

Proof. Without loss of generality, we can suppose that both $\mu_{1}$ and $\mu_{2}$ are finite. For any $E \in \mathfrak{B}$, we calculate the following double integral :

$$
I=\int C_{E}(x+y) d \mu_{1}(x) d \mu_{2}(y) .
$$

Since $I=\int \mu_{1}(E-y) d \mu_{2}(y)=\int \mu_{2}(E-x) d \mu_{1}(x)$ from Fubini's theorem, keeping the $X$-quasi-invariance of $\mu_{1}$ and $\mu_{2}$ in mind, we can see that $\mu_{1}(E)=0$ implies $I=0$ and thus $\mu_{2}(E)=0$. (q. e. d.)

Corollary. Suppose that $\mathfrak{B}$ satisfies the condition in Prop. 4.1 and that a measure $\mu$ defined on $\mathfrak{B}$ is $\sigma$-finite and $X$-quasi-inv. Then $\mu$ is quasi-inv. also with respect to homotheties. Namely, defining the expanded measure $\tau_{\lambda} \mu$ by the relation: " $\tau_{\lambda} \mu(E)=\mu(\lambda E)$ for all $E \in \mathfrak{B}^{\prime \prime}$, we have $\tau_{\lambda} \mu \sim \mu$ for any $\lambda>0$. 
Proof. Since $\tau_{\lambda} \mu$ is $X$-quasi-inv., Prop. 4.1 assures that $\tau_{\lambda} \mu \sim \mu$ 。 It is well known that if $X$ is finite dimensional, the condition in Prop. 4. 1 is fulfilled for the family of all Borel sets. Even in case of infinite dimension, this condition is fulfilled for $\mathfrak{B}_{R}$ provided that $R$ is a finite dimensional subspace of $X^{\prime}$, because $\mathfrak{B}_{R}$ can be identified with $\overline{\mathfrak{B}}_{R}$ as seen in $\S 2$.

For $n$-dimensional vector space $R^{n}$, we know that the Lebesgue measure is $R^{n}$-invariant. Hence any $\sigma$-finite and $R^{n}$-quasi-invariant measure $\mu$ is equivalent with the Lebesgue measure. Namely, it is written as $d \mu=f(x) d x$, where $d x$ means the Lebesgue measure and $f(x)$ is a Baire function such as $0<f(x)<\infty$ for almost all $x$. From this we get

$$
\frac{d \tau_{y} \mu}{d \mu}(x) \equiv \rho(y, x)=\frac{f(x+y)}{f(x)}
$$

Hence the following proposition;

Proposition 4.2. If $R$ is a finite dimensional subspace of $X^{\prime}$, then for any $\sigma$-finite and $X$-quasi-invariant measure $\mu$ on $\mathfrak{B}_{R}$ the density function $\frac{d \tau_{y} \mu}{d \mu}(x)=\rho(y, x)$ is $\mathfrak{B}_{R} \times \mathfrak{B}_{R}-$ measurable as a function of two variables.

\section{$\S 5$. Measurability of density function}

Proposition 5. 1. If $R\left(\subset X^{\prime}\right)$ is weakly separable and if a mesure $\mu$ on $\mathfrak{B}_{R}$ is finite and $X$-quasi-invariant, the density function $\frac{d \tau_{y} \mu}{d \mu}(x)$ $=\rho(y, x)$ is $\mathfrak{B}_{R} \times \mathfrak{B}_{R}-$ measurable as a function of two variables (with a suitable modification on a $\mu \times \mu$-null set).

Proof. The density function $\rho(y, x)$ is necessarily $\mathfrak{B}_{R}$-measurable as a function of $x$ for any fixed $y$. Let $\left\{R_{n}\right\}$ be of the same meaning in Prop. 3. 1, then by Prop. 3.2, putting $\frac{d \tau_{y} \mu_{n}}{d \mu_{n}}(x)=\rho_{n}(y, x)$, $\left\{\sqrt{\rho_{n}(y, x)}\right\}$ converges to $\sqrt{\rho(y, x)}$ in $L_{\mu}^{2}(X)$ for any fixed $y$.

As seen in Prop. 4.2, each $\sqrt{\rho_{n}(y, x)}$ is $\mathfrak{B}_{R} \times \mathfrak{B}_{R}$-measurable. Moreover we can see easily that

$$
N_{n, m}(y) \equiv\left\|\sqrt{\rho_{n}(y, x)}-\sqrt{\rho_{m}(y, x)}\right\|_{\mu_{(x)}} \leqq 2 \sqrt{\mu(X)},
$$


thus they are uniformly bounded as functions of $y$. Hence Lebesgue's theorem is applicable and it shows us that $\left\{\sqrt{\rho_{n}(y, x)}\right\}$ forms a Cauchy sequence in $L_{\mu \times \mu}^{2}(X \times X)$ as functions of two variables. Let $g(y, x)$ be the limit function of this Cauchy sequence. Then, a suitable subsequence of $\left\{\sqrt{\rho_{n}(y, x)}\right\}$ converges to $g(y, x)$ for $\mu \times \mu$-almost all $(y, x)$. From this, we see that $g(y, x)=\sqrt{\rho(y, x)}$ and hence $\rho(y, x)=(g(y, x))^{2}$ for $\mu \times \mu$-almost all $(y, x)$. (q. e. d.)

\section{$\S 6$. Construction of $X$-invariant measure}

In general, we can not say whether a given $G$-quasi-inv. measure $\mu$ has or has not an equivalent $G$-inv. measure. But if $G=X$ and if $\mu$ is a $\sigma$-finite measure on $\mathfrak{B}_{R}$ where $R$ is weakly separable, the answer is positive.

Proposition 6.1. If $R\left(\subset X^{\prime}\right)$ is weakly separable, then $\mathfrak{B}_{R}$ satisfies the condition in Prop. 4. 1, namely the mapping: $(x, y) \rightarrow x+y$ is a $\mathfrak{B}_{R}$-measurable mapping.

Proof. In case of a finite dimensional $R$, we have seen in $\S 4$ that this proposition is true. For an infinite dimensional $R$, we can prove it in a similar way as in the proof of Prop. 3.1.

Proposition 6.2. For a triplet $\{X, \mathfrak{B}, \mu\}$, assume the following three conditions:

1) $(x, y) \rightarrow x+y$ is a $\mathfrak{B}$-measurable mapping,

2) $\mu$ is $\sigma$-finite and $X$-quasi-invariant,

3) the density function $\rho(y, x)$ is $\mathfrak{B} \times \mathfrak{B}$-measurable (with a modification on a $\mu \times \mu-$ null set).

Then, we can construct an $X$-invariant, $\sigma$-finite measure $\mu_{0}$ which is equivalent with $\mu$.

Proof. The dersity function $\rho(y, x)$ satisfies the functional equation ;

$$
\rho(y+z, x)=\rho(y, x+z) \rho(z, x)
$$

(for all $y, z \in X$ and for $\mu$-almost all $x$ )

Except on a $\mu \times \mu$-null set, $\rho(y, x)$ is equal to $\mathfrak{B} \times \mathfrak{B}$-measurable function $\rho(y, x)$, hence 


$$
\begin{array}{ll}
\hat{\rho}(y+z, x)=\hat{\rho}(y, x+z) \hat{\rho}(z, x) & \text { (for } \mu \times \mu \times \mu \text {-almost all }(z, y, x)), \\
\text { and } 0<\hat{\rho}(y, x)<\infty & \text { (for } \mu \times \mu \text {-almost all }(y, x)) .
\end{array}
$$

Therefore, there exists $x_{0} \in X$ such that

$$
\begin{array}{ll}
\hat{\rho}\left(y+z, x_{0}\right)=\hat{\rho}\left(y, x_{0}+z\right) \hat{\rho}\left(z, x_{0}\right) & (\text { for } \mu \times \mu \text {-almost all }(y, z)), \\
0<\hat{\rho}\left(y, x_{0}\right)<\infty & \text { (for } \mu \text {-almost all } y) .
\end{array}
$$

Then $\hat{\rho}\left(y-x_{0}, x_{0}\right) \equiv f(y)$ is $\mathfrak{B}$-measurable, $0<f(y)<\infty$ for $\mu$ almost all $y$, and $\hat{\rho}(y, z)=\frac{f(y+z)}{f(z)}$ for $\mu \times \mu$-almost all $(y, z)$.

Put $\frac{1}{f(y)} d \mu(y)$ as $d \mu_{0}(y)$, then $\mu_{0}$ is $\sigma$-finite and $\mu \sim \mu_{0}$. Moreover, for $\mu$-almost all $y$.

$$
\begin{aligned}
\mu_{0}(E+y) & =\int_{E+y} \frac{1}{f(x)} d \mu(x)=\int_{E} \frac{1}{f(x+y)} d \tau_{y} \mu(x) \\
& =\int_{E} \frac{1}{f(x+y)} \frac{f(x+y)}{f(x)} d \mu(x)=\int_{E} \frac{1}{f(x)} d \mu(x)=\mu_{0}(E) .
\end{aligned}
$$

Thus $\mu_{0}$ is invariant under $\mu$-almost all translations. From this we see that $\mu_{0}$ is $X$-invariant as shown below. Put

$$
Y=\left\{y \in X \mid \tau_{y} \mu_{0}=\mu_{0}\right\},
$$

then i) $Y$ forms an additive group, and ii) ${ }^{\mathbb{B}} Z \in \mathfrak{B} ; \mu(Z)=0$ and $X=Y \cup Z$. Assume that $Y \neq X$, then ${ }^{3} x \in X ;(Y+x) \cap Y=\phi$, hence $Y+x \subset Z$ so that $Y \subset Z-x$, and $X=(Z-x) \cup Z$. Thus, since $\mu$ is $X$ quasi-inv., $\mu(X)$ must be zero which is a contradiction. (q.e.d.)

\section{$\S 7 . \quad$ Non-existence of $X$-quasi-invariant measure}

As last, we have reached the main theorem of this chapter.

Theorem. On a locally convex, infinite dimensional, topological vector space $X$, there does not exist any Borel measure which is $\sigma$-finite and $X$-quasi-invariant.

This theorem was proved by Sudakov, (c. f. Sudakov: [6]) but here we shall prove it in connexion with Weil's theorem.

For a locally convex, infinite dimensional, topological vector space $X$, its topological dual space $X^{*}$ is also infinite dimensional. 
And with respect to Borel measure, any linear continuous function on $X$ is measurable. So that, the following proposition is sufficient for the theorem.

Proposition 7.1. There exist no triplet $\{X, \mathfrak{B}, \mu\}$ such that;

i) $\mu$ is $\sigma$-finite and $X$-quasi-invariant,

ii) $\mathfrak{B} \supset \mathfrak{B}_{R}$ for some infinite dimensional subspace $R$ of $X^{\prime}$.

Proof. Assuming the existence of such a triplet, we shall show a contradiction occurs.

$R$ contains a subspace $R_{1}$ which is algebraically countable dimensional. Evidently $\mathfrak{B}_{R} \supset \mathfrak{B}_{R_{1}}$. Putting $\mu \mid \mathfrak{B}_{R_{1}}=\mu_{1}, \mu_{1}$ is an $X$ quasi-inv. measure defined on $\mathfrak{B}_{R_{1}}$, thus Prop. 5.1, 6.1, and 6.2 assure the existence of a measure $\mu_{0}$ which is $\sigma$-finite, $X$-invariant and $\mu_{0} \sim \mu_{1}$ on $\mathfrak{B}_{R_{1}}$.

As seen in $\S 2, \mu_{0}$ can be identified with a measure $\bar{\mu}_{0}$ on the factor space $X / R_{1}^{\perp}$. Then $X / R_{1}^{\perp}$ is infinite dimensional, and $\bar{\mu}_{0}$ is $X / R_{1}^{\perp}$-invariant.

From a general theory on topological vector spaces, we know that any locally precompact topological vector space is finite dimensional. So that we can reach a wanted contradiction, if $X / R_{1}^{\frac{1}{1}}$ becomes locally precompact with some topology. In order to construct such a topology, we use Weil's theorem. (c. f.: Weil [7]). At first, we formulate it for an additive group.

Proposition 7.2. (Weil's theorem) Let $X$ be an additive group and suppose that a triplet $\{X, \mathfrak{B}, \mu\}$ satisfies the following conditions.

i) $\mu$ is $\sigma$-finite and $X$-invariant,

ii) $(x, y) \rightarrow x-y$ is a $\mathfrak{B}-$ measurable mapping,

iii) $\{0\} \in \mathfrak{B}$,

iv) $\quad x \neq 0 \Rightarrow{ }^{a} E \in \mathfrak{B}, \quad 0<\mu(E)<\infty ; \mu((E+x) \ominus E)>0$.

Then, with some topology, $X$ becomes a locally precompact topological group.

Though we omit the proof here, we shall mention how to construct such a topology (Weil topology). Define a family $\mathbb{N}$ of subsets of $X$ by : 


$$
\begin{gathered}
N=\left\{N_{\varepsilon, E} \mid E \in \mathfrak{B}, 0<\mu(E)<\infty, 0<\varepsilon<2 \mu(E)\right\} \\
\text { where } \quad N_{\varepsilon, E}=\{x \in X \mid \mu((x+E) \ominus E)<\varepsilon\} .
\end{gathered}
$$

Then, choosing $N$ as a base of the system of neighbourhoods of 0 , we define Weil topology. With respect to this topology, $(x, y) \rightarrow x-y$ is continuous, and some precompact sets belong to $\mathbb{N}$. Putting $\boldsymbol{A}=\{E-E \mid E \in \mathfrak{B}, 0<\mu(E)<\infty\}, \mathbb{A}$ becomes another base of the system of neighbourhoods of 0 in Weil topology.

This Weil's theorem is kept valid for vector spaces with a few modifications.

Proposition 7.2'. Let $X$ be a real vector space and suppose that a triplet $\{X, \mathfrak{B}, \mu\}$ satisfies the following conditions,

i) $\mu$ is $\sigma$-finite and $X$-invariant,

ii) $(x, y) \rightarrow x+y$ is $\mathfrak{B}$-measurable,

ii) ${ }^{\prime} x \rightarrow \lambda x$ is $\mathfrak{B}$-measurable for real $\lambda \neq 0$,

iii) $\{0\} \in \mathfrak{B}$,

iv) $x \neq 0 \Rightarrow{ }^{\mathbb{B}} E \in \mathfrak{B}, 0<\mu(E)<\infty ;{ }^{\mathbb{B}} \varepsilon>0,{ }^{\nabla} \lambda \geqq 1, \mu((E+\lambda x) \ominus E)>\varepsilon$.

Then, using $N_{\varepsilon, E}$ of the previous proposition, if we define as $M_{\varepsilon, E}=\bigcup_{0 \leq \lambda \leq 1} \lambda N_{\varepsilon, E}$ and $\mathbb{M}=\left\{M_{\varepsilon, E}\right\}$, then $\mathbb{R}$ - becomes a base of the system of neighbourhoods of 0 in some topology which is compatible with algebraical structure of $X$ and makes $X$ locally precompact.

Proof. We can see easily that $M$ defines a topology which is weaker than Weil topology. We call this topology as M-topology. It fulfills the axiom of separation in virtue of the stronger condition iv).

The continuity of addition of two vectors with respect to $M-$ top. can be checked easily. Thus we should prove only the continuity of scalar multiplications, for which the following three conditions are sufficient. (c. f. Bourbaki; "Espaces vectoriels topologiques" page 7)
a) $M \in M \Rightarrow V|\lambda| \leqq 1 ; \lambda M \subset M$.
b) ${ }^{v} M \in M ; M$ is absorbing, namely $\bigcup_{0<\lambda} \lambda M=X$,
c) $M \in M \Rightarrow{ }^{V} \lambda>0,{ }^{\exists} M^{\prime} \in M ; \lambda M \supset M^{\prime}$. 
a) is evident from the definition of $M_{\varepsilon, E}$ and the symmetricity of $N_{\varepsilon, E}$.

At next, we can show b) is satisfied even for $\mathbb{N}$. Suppose that $N \in \mathbb{N}$ and $\bigcup_{0<\lambda} \lambda N \neq X$. From the equivalence of $\mathbb{N}$ and $\mathbb{A}$, we have ${ }^{g} E \in \mathfrak{B}, 0<\mu(E)<\infty ; E-E \subset N$. Thus $x \notin \bigcup_{0<\lambda} \lambda N$ implies $(E+\lambda x) \cap$ $\left(E+\lambda^{\prime} x\right)=\phi$ for $\lambda \neq \lambda^{\prime}$, Being $\mu(E)>0$, this is a contradiction against the $\sigma$-finiteness of $\mu$.

c) is proved also using the equivalence of $\mathbb{N}$ and $\mathbb{A}$, and the fact that $\mu(E)>0 \Rightarrow \mu(\lambda E)>0$.

Finally, we shall show that $X$ is locally precompact with respect to $\mathbb{M}$-topology. From Weil's theorem, some $N(\in \mathbb{N})$ is precompact with respect to Weil top., hence also with respect to $M$-top. Thus $M=\underset{0 \leqq \lambda \leqq 1}{\bigcup} \lambda N$ is precompact with respect to $M-$ top., for it is a continuous image of $[0,1] \times N$. It shows us the local precompactness of $X$. (q. e.d.)

We shall return to the proof of Prop. 7.1. Since $R_{1}(\subset R)$ is algebraically countable dimensional, the triplet $\left\{X / R_{1}^{i}, \overline{\mathfrak{B}}_{R_{1}}, \bar{\mu}_{0}\right\}$ evidently fulfills the conditions i) iv) of Prop. 7.2'. Thus $X / R_{1}^{\perp}$ becomes a locally precompact vector space with some topology. This contradicts against the infinite dimensionality of $X / R_{1}^{\perp}$, so that Prop. 7.1, hence the main theorem also, has been proved.

\section{Chapter II. Minlos' theorem}

Contents : Let $\chi(\xi)$ be a positive definite function defined on a real topological vector space $L$. In order that there exists corresponding meastre $\mu$ on the dual space $L^{*}$, the continuity of $\chi(\xi)$ is

a) recessary, if $L$ is metrizable,

b) sufficient, if $L$ is nuclear.

\section{§. Measure $\rightarrow$ Characteristic function}

Let $L$ be a real topological vector space, and $L^{*}$ be its dual space. Following $\S 2$, we can define the $\sigma$-ring $\mathfrak{B}_{L}$ of subsets of $L^{*}$, regarding $L$ as a subspace of $L^{* *}$. Then, for any $\xi \in L, \xi(x)$ is a $\mathfrak{B}_{L_{L}}$-measurable function. 
For a finite measure $\mu$ on $\mathfrak{B}_{L}$, we define the characteristic function $\chi(\xi)$ of $\mu$ as follows :

$$
\chi(\xi)=\int \exp (i \xi(x)) d \mu(x)
$$

Proposition 8.1. Properties of the characteristic function.

1) $\chi(\xi)$ is positive definite on $L$; namely for any finite set $\left\{\alpha_{i}\right\}$ of complex numbers, and for any finite subset $\left\{\xi_{i}\right\}$ of $L$,

$$
\sum_{i, j} \alpha_{i} \bar{\alpha}_{j} \chi\left(\xi_{i}-\xi_{j}\right) \geqq 0
$$

2) $\chi(0)=\mu\left(L^{*}\right)$

3) If a sequence $\left\{\xi_{n}\right\}$ converges to 0 weakly, then $\chi\left(\xi_{n}\right)$ converges to $\mu\left(L^{*}\right)$. Namely, $\chi(\xi)$ is weakly sequentially continuous.

Proof. 1) The left hand side of (2) can be written as

$$
\int\left|\sum_{j} \alpha_{j} \exp \left(i \xi_{j}(x)\right)\right|^{2} d \mu(x), \text { so that } \geqq 0 \text {. }
$$

2 ) is evident. 3) is proved by virtue of Lebesgue's theorem.

Corollary. If $L$ is metrizable, $\chi(\xi)$ is strongly continuous.

Proof. Since $\chi(\xi)$ is weakly sequentially cont., it must be strongly sequentially cont. However, on a metrizable space, any sequentially continuous function is continuous.

\section{$\S 9$. Characteristic function $\rightarrow$ Measure}

From now on, we suppose that $\chi(\xi)$ has been given, and try to construct the corresponding measure $\mu$.

Problem A. Suppose that a function $\chi(\xi)$ on a real topological vector space $L$ satisfies the following conditions:

a) $\chi(\xi)$ is positive definite on $L$,

b) $\chi(0)=1$,

c) $\chi(\xi)$ is continuous.

Then, on the dual space $L^{*}$, does there exist uniquely a measure $\mu$ whose characteristic function is $\chi(\xi)$ ?

In the case that $L$ is finite dimensional, Problem A was solved 
affirmatively by the classical Bochner's theorem. If $L$ is infinite dimensional, for a finite dimensional subspace $R$ of $L$, the restriction of $\chi(\xi)$ on $R$ is evidently fulfills the conditions a) c) (but positive definite on $R$ instead on $L$ ). So, the classical Bochner's theorem assures that there exists a triplet $\left\{L^{*}, \mathfrak{B}_{R}, \mu_{R}\right\}$ such that $\chi(\xi) \mid R$ becomes the characteristic function of $\mu_{R}$.

Proposition 9.1. 1) Let $R, R^{\prime}$ be two finite dimensional subspaces of $L$, then, $\mu_{R}\left|\mathfrak{B}_{R} \cap \mathfrak{B}_{R^{\prime}}=\mu_{R^{\prime}}\right| \mathfrak{B}_{R} \cap \mathfrak{B}_{R^{\prime}}$.

2) For any $R, \mu_{R}\left(L^{*}\right)=1$.

3) For any $\varepsilon>0$, there exists a neighbourhood $U$ of 0 in $L$ such that

$$
\xi \in U \Rightarrow \mu_{\{\xi\}}(\{x \in X|| \xi(x) \mid \geqq 1\})<\varepsilon .
$$

Here $\{\xi\}$ means the one-dimensional subspace of $L$ which is generated from $\xi$.

Proof. 1), 2), and 3) can be proved respectively from the uniqueness part of the classical Bochner's theorem, from $\chi(0)=1$, and from the continuity of $\chi(\xi)$.

From Prop. 9.1 we get the following proposition.

Proposition 9.2. If a function $\chi(\varepsilon)$ on $L$ satisfies the conditions a) c) of Problem $A$, it becomes the characteristic function of a finitely additive measure $\mu$ on $\mathfrak{R}_{L} \equiv \bigcup_{R \subset L} \mathfrak{B}_{R}$ and the following conditions are satisfied.

(i) For any fixed $R, \mu$ is completely additive on $\mathfrak{B}_{R}$,

(ii) $\mu\left(L^{*}\right)=1$,

(iii) ${ }^{V} \varepsilon>0,{ }^{3} U$ (neighbourhood of 0 in $L$ )

$$
\xi \in U \Rightarrow \mu(|\xi(x)| \geqq 1)<\varepsilon .
$$

At next, we consider the converse.

Proposition 9. 3. Suppose that on the finite-ring $\mathfrak{R}_{L} \equiv \bigcup_{R \subset L} \mathfrak{B}_{R}$, a finitely additive measure $\mu$ is given and satisfies the conditions (i) (iii) of the previous proposition. If we define $\chi(\xi)$ by

$$
\chi(\xi)=\int \exp (i \xi(x)) d \mu(x),
$$


it fulfills the conditions $a) \sim c$ ) of Problem $A$.

Proof is easy.

Thus, Problem A is equivalent with the following Problem B.

Problem B. Can we extend uniquely a finitely additive measure $\mu$ on $\Re_{L}$ to a completely additive measure on $\mathfrak{B}_{L}$, under the conditions (i) (iii) of Prop. 9.2?

Problem A follows the formulation by Bochner, while Problem B follows the formulation by Minlos.

Proposition 9.4. Uniqueness part of Problem $B$ is answered always affirmatively.

Proof. Suppose that $\mu$ can be extended to two completely additive measures $\mu_{1}$ and $\mu_{2}$ on $\mathfrak{B}_{L}$. Let $\mathfrak{A}$ be the family of all sets $E$ which satisfy $\mu_{1}(E)=\mu_{2}(E)$. Evidently $\mathfrak{A}$ forms a $\sigma$-ring and $\mathfrak{A} \supset \mathfrak{R}_{L}$, hence $\mathfrak{A} \supset \mathfrak{B}_{L}$, because $\mathfrak{B}_{L}$ is the smallest $\sigma$-ring which includes the finite-ring $\Omega_{L}$. (q. e.d.)

\section{$\S 10$. Conditions for extendability}

Next, we shall discuss the extendability part of Problem B.

Proposition 10.1. In order that a finitely additive finite measure $\mu$ defined on a finite-ring $\Omega$ can be extended to a completely additive measure on $\mathfrak{B}$ (=the smallest $\sigma$-ring which contains $\Re$ ), it is necessary and sufficient that;

$$
\begin{gathered}
A_{i} \in \Re, \quad A_{1} \supseteqq A_{2} \supseteqq \cdots \supseteqq A_{n} \supseteqq \cdots \\
\lim _{n} \mu\left(A_{n}\right) \neq 0 \Rightarrow \bigwedge_{n} A_{n} \neq \phi .
\end{gathered}
$$

Proof is omitted here, for this is a general theorem in measure theory.

Proposition 10.2. In order that Prob. B is solved affirmatively, the following condition is sufficient.

$$
\begin{gathered}
\left.{ }^{\vee} \varepsilon>0,{ }^{{ }^{B} C} \quad \text { (weakly compact set in } L^{*}\right) \\
{ }^{\forall} A \in \Re_{L}, A \cap C=\phi \Rightarrow \mu(A)<\varepsilon .
\end{gathered}
$$


Proof. Suppose that $A_{i} \in \mathfrak{R}_{L}, A_{1} \supseteqq A_{2} \supseteqq \cdots \supseteqq A_{n} \supseteqq \cdots$ and $\lim _{n} \mu\left(A_{n}\right)$ $=2 \alpha>0$. For any finite dimensional subspace $R$ of $L, \mu \mid \mathfrak{K}_{R}$ is completely additive from the condition (i), and it can be identified with a measure on the factor space $L^{*} / R^{\perp}$. Since any Borel measure on a finite dimensional vector space is topologically regular (i.e. $\mu(A)=\sup _{\substack{F \subset A \\ F \text { :closed }}} \mu(F)$ for any Borel set $A$ ), we can choose such a sequence $\left\{F_{n}\right\}$ of weakly closed cylinder sets that satisfies :

$$
\begin{gathered}
F_{i} \in \Omega_{L}, F_{1} \supseteqq F_{2} \supseteqq \cdots \supseteqq F_{n} \supseteqq \cdots \\
{ } n: F_{n} \cong A_{n} \text { and } \lim _{n} \mu\left(F_{n}\right) \geqq \alpha>0 .
\end{gathered}
$$

Thus, we should show only that $\bigcap_{n} F_{n} \neq \phi$.

If the condition of Prop. 10.2 is fulfilled, there exists a weakly compact set $C$ such that

$$
{ }^{v} A \in \Re_{L}, A \cap C=\phi \Rightarrow \mu(A)<\alpha .
$$

So that $\mu\left(F_{n}\right) \geqq \alpha$ implies $F_{n} \cap C \neq \phi$. Since any decreasing sequence of non-empty compact sets has non-empty intersection, we get $\bigcap_{n}\left(F_{n} \cap C\right) \neq \phi$, hence $\bigcap_{n} F_{n} \neq \phi$. (q. e. d.)

Let $U$ be a neighbourhood of 0 in $L$ and define the polar set $\stackrel{\circ}{U}$ of $U$ by the relation : $\dot{U}^{\circ}=\left\{x \in L^{*}|| \xi(x) \mid \leqq 1\right.$ for $\left.{ }^{v} \xi \in U\right\}$.

From the general theory of topological vector spaces, we know that $\stackrel{\circ}{U}$ is weakly compact. Thus, from Prop. 10.2, we get:

Proposition 10.2'. In order that Problem $B$ is solved affirmatively, the following condition is sufficient.

$$
\begin{gathered}
\left.{ }^{V} \varepsilon>0,{ }^{\exists} U \text { (neighbourhood of } 0 \text { in } L\right) \\
{ }^{V} A \in \mathfrak{I}_{L}, A \cap \stackrel{\circ}{U}=\phi \Rightarrow \mu(A)<\varepsilon .
\end{gathered}
$$

We have not yet taken into account of the contiuuity of $\mu$ (namely, the condition (iii) of Prop. 9.2). Here, we shall discuss the relation between the condition (iii) and Prop. 10.2'.

Proposition 10.3. In order that Problem $B$ is solved affirmatively, the following condition is sufficient. (Later, we shall quote it as the condition $(\mathrm{SC})$ ). 
$(\mathbf{S C}):{ }^{{ }^{a}} \eta(\varepsilon)$ : increasing function which is defined for positive small numbers and $\lim _{\varepsilon \rightarrow+0} \eta(\varepsilon)=0$,

$\checkmark U:$ (neighbourhood of 0 in $L)$,

${ }^{g} V$ : (convex, symmetric neighbourhood of 0 in $\left.L\right)$,

${ }^{\forall} R$ : (finite dimensional subspace of $L$ );

If $\mu(|\xi(x)| \geqq 1)<\varepsilon$ for $\quad \forall \xi \in U \cap R$, then $\mu\left((V \cap R)^{\circ}\right) \geqq 1-\eta(\varepsilon)$.

Proof. The condition (iii) of Prop. 9.2 assures that

$$
\begin{gathered}
\left.{ }^{\vee} \varepsilon>0,{ }^{\exists} U \text { (neighbourhood of } 0 \text { in } L\right) \\
\mu(|\xi(x)| \geqq 1)<\varepsilon \text { for }{ }^{\nabla} \xi \in U .
\end{gathered}
$$

Thus, if the condition (SC) is fulfilled,

${ }^{g} V$ (convex, symmetric neighbourhood of 0 in $L$ ), ${ }^{\forall} R \subset L$;

$$
\mu\left((V \cap R)^{\circ}\right) \geqq 1-\eta(\varepsilon) \text {. }
$$

Therefore, in order to show that the sufficient condition of Prop. 10.2' is fulfilled, we should only show

$$
{ }^{\mathrm{V}} A \in \mathfrak{B}_{R}, \quad A \cap \stackrel{\circ}{V}=\phi \Rightarrow A \cap(V \cap R)^{\circ}=\phi .
$$

(Since $(3)$ implies $\left.\mu(A) \leqq 1-\mu(V \cap R)^{\circ}\right) \leqq 1-(1-\eta(\varepsilon))=\eta(\varepsilon)$ and we can choose $\eta(\varepsilon)$ arbitrarily small by taking $\varepsilon$ sufficiently small.)

Proof of (3). We shall prove the contraposition of (3).

Suppose that $A \cap(V \cap R)^{\circ} \neq \phi$. If $x \in A$ and $x \in(V \cap R)^{\circ}$, then $|\xi(x)| \leqq 1$ for any $\xi \in V \cap R$. Since $V$ is convex and symmetric, Hahn-Banach's theorem shows us that;

$$
\begin{aligned}
& { }^{\exists} x^{\prime} \in L^{*} ;\left|\xi\left(x^{\prime}\right)\right| \leqq 1 \text { for } \quad v \xi \in V \\
& \text { and } \xi(x)=\xi\left(x^{\prime}\right) \text { for } \quad v \xi \in R
\end{aligned}
$$

Evidently $x^{\prime} \in \stackrel{\circ}{V}$. On the other hand, $x-x^{\prime} \in R^{\perp}$ and $x \in A$ imply that $x^{\prime} \in A\left(\in \mathfrak{B}_{R}\right)$. Hence, $x^{\prime} \in A \cap \stackrel{\circ}{ }$ so that $A \cap \stackrel{\circ}{V} \neq \phi$. (q. e. d.)

\section{$\S 11$. Definition and some properties of nuclear spaces}

(For details, c. f. Grothendieck : [8], and Mityagin : [9]).

Though there are many diferent definitions of the nuclearity, 
we adopt the following one, for it seems intuitional to the author.

Let $L$ be an infinite dimensional, locally convex vector space, and let $\mathfrak{U}$ be the system of convex and symmetric neighbourhoods of 0 in $L$.

Definition 11.1. L is said to be nuclear, if it satisfies;

$$
{ }^{v} U \in \mathfrak{U},{ }^{\mathbb{a}} V \in \mathfrak{M},{ }^{\vee} n \text { (positive integer), }
$$

${ }^{\pi} E_{n} \quad(n$-dimensional subspace of $L) ; V \subset E_{n}+\frac{1}{n} U$.

Proposition 11.1. The following two conditions are equivalent,

a) ${ }^{g} \lambda_{0}>0,{ }^{\vee} U \in \mathfrak{U},{ }^{\exists} V \in \mathfrak{U},{ }^{v} n,{ }^{g} E_{n} ; V \subset E_{n}+\frac{1}{n^{\lambda_{0}}} U$,

b) ${ }^{v} \lambda>0, \quad{ }^{v} U \in \mathfrak{U},{ }^{g} V \in \mathfrak{U},{ }^{v} n,{ }^{a} E_{n} ; V \subset E_{n}+\frac{1}{n^{\lambda}} U$.

Proof is omitted.

Definition 11.1'. L is said to be nuclear, if it satisfies a) or b) of Prop. 11.1.

(Note: Especially, in Def. 11.1, we have taken $\lambda=1$.)

Proposition 11.2. An infinite dimensional, locally convex vector space $L$ is nuclear, if and only if it satisfies the following condition:

${ }^{v} U \in \mathfrak{H},{ }^{a} V \in \mathfrak{H}$,

${ }^{a} x_{i} \in L^{*},{ }^{a} \xi_{i} \in L$ for $i=1,2, \cdots$, such that

$$
\sum_{i=1}^{\infty}\left|x_{i}\right|_{V}^{\prime}\left|\xi_{i}\right|_{U}<\infty \text { and for } \quad \forall \xi \in L,\left|\xi-\sum_{i=1}^{n} x_{i}(\xi) \xi_{i}\right|_{U} \rightarrow 0 \text {. }
$$

Here, we put $|\xi|_{U} \equiv \inf _{\lambda}\left\{\lambda>0 \mid \frac{1}{\lambda} \xi \in U\right\}$ and $|x|_{V}^{\prime} \equiv \sup _{\xi \in V}|x(\xi)|$.

Proof is omitted.

Proposition 11.3. The topology of a nuclear space $L$ can be defined by a family of Hilbertian semi-norms, and $L$ is separable with respect to each of these semi-norms.

Proof. In Prop. 11.2, without loss of generality we can assume that $\left|x_{i}\right|_{V}^{\prime}=\left|\xi_{i}\right|_{U}$ for all $i$. Then,

$$
\sum_{i=1}^{\infty}\left|x_{i}\right|_{V}^{\prime}\left|\xi_{i}\right|_{U}=\sum_{i=1}^{\infty}\left|x_{i}\right|_{V}^{\prime 2}=\sum_{i=1}^{\infty}\left|\xi_{i}\right|_{U}^{2} \quad\left(=M^{2}: \text { put }\right)<\infty .
$$


For $\xi, \eta \in L$, the bilinear form $\langle\xi, \eta\rangle_{H} \equiv \sum_{i=1}^{\infty} x_{i}(\xi) x_{i}(\eta)$ satisfies the properties of an inner product except the condition: $"\langle\xi, \xi\rangle_{H}=0 \Rightarrow$ $\xi=0$ ". Hence $\|\xi\|_{H}=\sqrt{\sum_{i=1}^{\infty} x_{i}(\xi)^{2}}$ is a Hilbertian semi-norm. Evidently,

$$
\|\xi\|_{H}^{2} \leqq \sum_{i=1}^{\infty}|\xi|_{V}^{2}\left|x_{i}\right|_{V}^{\prime 2}=M^{2}|\xi|_{V}^{2}
$$

while $\quad|\xi|_{U} \leqq \sum_{i=1}^{\infty}\left|x_{i}(\xi)\right|\left|\xi_{i}\right|_{U} \leqq \sqrt{\sum_{i=1}^{\infty} x_{i}(\xi)^{2}} \sqrt{\sum_{i=1}^{\infty}\left|\xi_{i}\right|_{U}^{2}}=M\|\xi\|_{H}$.

Thus we get $\frac{1}{M}|\xi|_{U} \leqq\|\xi\|_{, H} \leqq M|\xi|_{V}$.

This is sufficient for Prop. 11.3, since we can define $\|\xi\|_{H}$ for any $U \in \mathfrak{l}$.

Proposition 11.4. Suppose that the topology of an infinite dimensional vector space $L$ is defined by a family of Hilbertian semi-norms and $L$ is separable with respect to each of these semi-norms. Then for the nuclearity of $L$, the following condition is necessary and sufficient.

$v\|\|_{H}$ (Hilbertian semi-norm),

${ }^{3} V \quad$ (neighbourhood of 0 in $L$ ),

${ }^{\sharp}\left\{\xi_{i}\right\} \quad$ (complete orthonormal system in $\left.\|\cdot\|_{H}\right)$,

${ }^{3} a_{i}>0$ such that $\sum_{i=1}^{\infty} a_{\imath}^{2}<\infty$;

$$
V \subset\left\{\xi \in L \mid \sum_{i=1}^{\infty} \frac{\left\langle\xi, \xi_{i}\right\rangle_{H}^{2}}{a_{i}^{2}} \leqq 1\right\}
$$

(Namely, $V$ is contained in an ellipsoid with square summable principal axis in $\|\cdot\|_{H^{-}}$semi-norm.)

Proof is omitted.

Proposition 11.5. A nuclear space $L$ is necessarily a Montel space (i.e. any bounded subset of $L$ is totally bounded).

Proof. Let $B$ be a bounded subset of $L$. It is sufficient to show ; ${ }^{V} U$ (neighbourhood of 0 in $L$ ),

${ }^{\sharp} n \quad$ (positive integer), $\quad{ }^{\exists} \xi_{i} \in L \quad(i=1,2, \cdots, n)$ 


$$
B \subset \bigcup_{i=1}^{n}\left(U+\xi_{i}\right) .
$$

Since $L$ is nuclear, there exists a neighbourhood $V$ of 0 that is totally bounded with respect to the semi-norm $|\cdot|_{U}$. Namely,

$$
{ }^{V} \lambda>0,{ }^{a} n^{\prime},{ }^{a} \xi_{i}^{\prime} \in L ; V \subset \bigcup_{i=1}^{n^{\prime}}\left(\lambda U+\xi_{i}^{\prime}\right) .
$$

On the other hand, $B$ being a bounded set, we have $\lambda B \subset V$ for some $\lambda>0$. Hence, $B \subset \bigcup_{i=1}^{n^{\prime}}\left(U+\frac{1}{\lambda} \xi_{i}^{\prime}\right)$. (q. e. d.)

Example. Schwartz's function spaces $(\mathscr{Y})$ and (D) are nuclear.

In $(\varphi)$, for instance, consider a neighbourhood $U$ of 0 ;

$$
U=\left\{\xi(t)|| \xi(t) \mid \leqq 1 \text { for } \quad \forall t \in R^{m}\right\} .
$$

Then, choose $V$ as;

$$
\begin{array}{r}
V=\left\{\xi(t) \mid \operatorname{Max}\left(|\xi(t)|,\left|\frac{\partial \xi(t)}{\partial t_{1}}\right|, \cdots,\left|\frac{\partial \xi(t)}{\partial t_{m}}\right|,|t|^{2}|\xi(t)|\right) \leqq 1\right. \\
\text { for } \left.\quad v t \in R^{m}\right\} .
\end{array}
$$

It is evident that :

(i) $\xi(t) \in V \Rightarrow|\xi(t)| \leqq \frac{1}{n}$ for $|t| \geqq \sqrt{n}$.

(ii) $\xi(t), \eta(t) \in V, \xi\left(t_{0}\right)=\eta\left(t_{0}\right)$

$$
\Rightarrow|\xi(t)-\eta(t)| \leqq \frac{1}{n} \quad \text { for } \quad\left|t-t_{0}\right| \leqq \frac{1}{2 n} .
$$

Thus, if we cover the ball $B=\{t|| t \mid \leqq \sqrt{n}\}$ with a family of small balls $B_{j}$ with radius $\frac{1}{2 n}$ and center $t_{j}$, then $\xi\left(t_{j}\right)=\eta\left(t_{j}\right)$ for $v_{j}$ implies that $\xi(t)-\eta(t) \in \frac{1}{n} U$.

Let $1=\sum \alpha_{j}(t)$ be a decomposition of unity such that Car. $\left(\alpha_{j}\right) \subset B_{j}$. Then, $V$ can be approximated within $\frac{1}{n} U$ by linear combinations of $\alpha_{j}(t)$. Since the number of the small balls $B_{j}$ is of 
the order of $n^{m / 2}\left(\frac{1}{2 n}\right)^{-m}=2^{m} n^{3 m / 2},\left\{\alpha_{j}(t)\right\}$ generates a $2^{m} n^{3 m / 2}$-dimensional subspace of $(\varphi)$. Thus, $V$ can be approximated within $\frac{1}{n} U$ by a $2^{m} n^{3 m / 2}$-dimensional subspace, hence within $k^{-2 / 3 m} U$ by a $k-$ dimensional subspace. This is just our definition of the nuclearity. Even if we consider a more complicated (i.e. dependent on derivatives of higher-crders) neighbourhood as $U$, we can construct the corresponding $V$ in a similar way. Thus, $(\varphi)$ is nuclear.

Also the nuclearity of (D) can be proved in a similar way.

\section{§12. Minlos' theorem (Bochner's theorem)}

Theorem A (Bochner's theorem)

In case that $L$ is nuclear, Problem $A$ in $\S 9$ is solved affirmatively.

\section{Theorem B (Minlos' theorem)}

In case that $L$ is nuclear. Problem $B$ in $\$ 9$ is solved affirmatively.

Proof. Two theorems being equivalent as mentioned in $\S 9$, here we shall prove Minlos' theorem. In order to check the condition (SC) of Prop. 10.3, we shall use the following fundamental lemma.

Fundamental lemma. Let $R^{N}$ be an $N$-dimensional vector space and $R^{\prime N}$ be its dual space. Namely,

$$
\begin{aligned}
R^{N} & =\left\{x=\left(x_{1}, \cdots, x_{N}\right) \mid x_{i} ; \text { real }\right\}, \\
R^{\prime N} & =\left\{\xi=\left(\xi_{1}, \cdots, \xi_{N}\right) \mid \xi_{i} ; \text { real }\right\} \text { and } \xi(x)=\sum_{i=1}^{N} \xi_{i} x_{i} .
\end{aligned}
$$

Let $S$ be the unit ball in $R^{N}$, and $F$ be an ellipsoid in $R^{\prime N}$.

$$
S=\left\{x \in R^{N} \mid \sum_{k=1}^{N} x_{k}^{2} \leqq 1\right\}, \quad F=\left\{\xi \in R^{\prime N} \mid \sum_{k=1}^{N} b_{k}^{2} \xi_{k}^{2} \leqq 1\right\} .
$$

Then, for any (probability) Borel measure $\mu$ on $R^{N}$, the following condition a) implies the condition b). 
a) $\xi \in F \Rightarrow \mu(\{x \mid \xi(x) \geqq 1\})<\varepsilon$.

b) $\mu(S) \geqq 1-\gamma_{0}(\varepsilon+D)$ where $D=\sum_{k=1}^{N} b_{k}^{2}$.

Here $\gamma_{0} \doteqdot 6.4$ is an absolute constant which does not depend on $N$.

Proof of this lemma will be given in the next section.

From this lemma, we can show that the condition $(S C)$ is fulfilled in case that $L$ is nuclear. Let $L$ be nuclear, and $\|\cdot\|_{H}$ be a continuous Hilbertian semi-norm on $L$. From Prop. 11.4,

${ }^{\sharp} V \quad$ (neighbourhood of 0 in $L$ ),

${ }^{3}\left\{\xi_{i}\right\}$ (complete orthonormal system of $L$ in $\|\cdot\|_{H}$ ),

$\exists\left\{a_{i}\right\} \quad$ (sequence of positive numbers),

$$
\begin{aligned}
& \text { such that } \sum_{i=1}^{\infty} a_{i}^{2}\left(=D_{0} ; \text { put }\right)<\infty \\
& V \subset\left\{\xi \in L \mid \sum_{i=1}^{\infty} \frac{\left\langle\xi, \xi_{i}\right\rangle_{H}^{2}}{a_{i}^{2}} \leqq 1\right\} \equiv W
\end{aligned}
$$

Without loss of generality, for any given $\varepsilon>0$, we can assume that $D_{0}<\varepsilon$.

Let $R$ be a finite dimensional subspace of $L$. Putting $U=\left\{\xi \in L \mid\|\xi\|_{H} \leqq 1\right\}, U \cap R$ is the unit ball in $R$. On the other hand, $V \cap R \subset W \cap R$, and $W \cap R$ is an ellipsoid in $R$. So that there exists a complete orthonormal system $\left\{\xi_{i}^{\prime}\right\}$ of $R$ (in $\|\cdot\|_{H}$ ), such that;

$$
W \cap R=\left\{\xi \in R \mid \sum_{i=1}^{N} \frac{\left\langle\xi, \xi_{i}^{\prime}\right\rangle_{H}^{2}}{b_{i}^{2}} \leqq 1\right\},
$$

where $N$ is the dimersion of $R$, and $\sum_{i=1}^{N} b_{i}^{2} \leqq D_{0}<\varepsilon$.

Put $\frac{\left\langle\xi, \xi_{i}^{\prime}\right\rangle_{H}}{b_{i}}=\bar{\xi}_{i}$, and regard $\bar{\xi}_{i}$ as the coordinates of $\xi$. Then,

$$
\begin{array}{r}
U \cap R=\left\{\xi \in R \mid \sum_{i=1}^{N} b_{i}^{2} \xi_{i}^{2} \leqq 1\right\}, \\
\text { while } W \cap R=\left\{\xi \in R \mid \sum_{i=1}^{N} \xi_{i}^{2} \leqq 1\right\} .
\end{array}
$$

Let $R^{\prime}$ be the dual space of $R$. It is isomorphic with $L^{*} / R^{\perp}$, so we identify them. Evidently, $(W \cap R)^{\circ} \subset(V \cap \mathrm{R})^{\circ}$ and

$$
(W \cap R)^{0}=\left\{\bar{x} \in R^{\prime} \mid \sum_{i=1}^{N} \bar{x}_{i}^{2} \leqq 1\right\}
$$


where $\bar{x}_{i}$ is the canonical coordinates of $\xi_{i}$.

Let $\mu$ be a finitely additive measure which satisfies the conditions (i) (iii) of Prop. 9.2. Then, $\mu \mid \mathfrak{B}_{R}$ is a measure on $L^{*} / R^{\perp} \simeq R^{\prime}$. Suppose that $\mu(|\xi(x)| \geqq 1)<\varepsilon$ for $\forall \xi \in U \cap R$, then from the fundamental lemma, we get

$$
\begin{aligned}
\mu\left(\left\{x \mid \sum_{i=1}^{N} \bar{x}_{i}^{2} \leqq 1\right\}\right) & =\mu\left((W \cap R)^{\circ}\right) \\
& \geqq 1-\gamma_{0}\left(\varepsilon+D_{0}\right) \geqq 1-2 \gamma_{0} \varepsilon .
\end{aligned}
$$

This shows us that condition (SC) of Prop. 10.3 is fulfilled by choosing $\eta(\varepsilon)=2 \gamma_{0} \varepsilon_{\text {。 }} \quad$ (q. e. d.)

Thus we have solved Problem B affirmatively under the condition that $L$ is nuclear. Conversely, this condition is necessary in the following sense.

Proposition 12.1. In the case that the topology of a locally convex vector space $L$ is defined by a family of countable Hilbertian norms, if $L$ is not nuclear, a suitable Gaussian measure can not be extended to a completely additive one.

Proof is omitted. (c. f. Minlos : [10])

\section{$\S 13$. Proof of fundamental lemma}

Let $R^{N}$ be an $N$-dimensional vector space, and $\mu$ be a probability Borel measure on $R^{N}$, which satisfies the condition a) in the fundamental lemma.

Let $m$ be another Borel measure on $R^{\prime N}$, which satisfies the following conditions :

i) $m\left(R^{\prime N}\right)=1$,

ii) rotationally invariant, i.e. for any rotation $u$ of $R^{\prime N}$, $m(E)=m(u E)$,

iii) $\int \xi_{i}^{2} d m(\xi)=1$ for ${ }^{v} i$.

Consider the product measure $\mu \times m$ on $R^{N} \times R^{\prime N}$, and calculate the double integral;

$$
I \equiv \int_{\xi(x) \geqq 1} d \mu(x) d m(\xi) .
$$


Putting ;

$$
F=\left\{\xi \in R^{\prime N} \mid \sum_{k=1}^{N} b_{k}^{2} \xi_{k}^{2} \leqq 1\right\}
$$

we have

$$
\begin{gathered}
\int_{\xi(x) \geqq 1} d \mu(x)==\mu(\{x \mid \xi(x) \geqq 1\})\left\{\begin{array}{ccc}
<\varepsilon & \text { if } & \xi \in F \\
\leqq 1 & \text { if } & \xi \notin F .
\end{array}\right. \\
\therefore \quad I \leqq \varepsilon m(F)+1-m(F) \leqq \varepsilon+(1-m(F)) .
\end{gathered}
$$

On the other hand, since $m$ is rotationally invariant,

$$
\int_{\xi(x) \geqq 1} d m(\xi)=\int_{\xi_{1} \geqq 1 /|x|} d m(\xi) .
$$

The right hand side is an increasing function of $|x|=\sqrt{\sum_{k=1}^{N} x_{k}^{2}}$, so that

$$
\int_{\xi(\lambda) \geqq 1} d m(\xi) \begin{cases}\geqq 0 & \text { for } \quad x \in S, \\ \geqq \int_{\xi_{1} \geqq 1} d m(\xi) & \text { for } \quad x \notin S,\end{cases}
$$

where $S$ is the unit ball in $R^{N}$.

Thus, we get;

$$
I \geqq \int_{\xi_{1} \geqq 1} d m(\xi) \times(1-\mu(S)) .
$$

Comparing (5) with (6), it is easily seen that

$$
1-\mu(S) \leqq(\varepsilon+1-m(F))\left[\int_{\xi_{1} \geqq 1} d m(\xi)\right]^{-1} .
$$

At next, we shall estimate $m(F)$. From (4), we get

$$
\sum_{k=1}^{N} b_{k}^{2} \xi_{k}^{2} \begin{cases}\geqq 0 & \text { for } \quad \xi \in F \\ \geqq 1 & \text { for } \quad \xi \notin F,\end{cases}
$$

so that considering the condition iii),

$$
1-m(F) \leqq \int \sum_{k=1}^{N} b_{k}^{2} \xi_{k}^{2} d m(\xi)=\sum_{k=1}^{N} b_{k}^{2} \quad(\equiv D) .
$$

Thus, (7) becomes; 


$$
1-\mu(S) \leqq(\varepsilon+D)\left[\int_{\xi_{1} \geqq 1} d m(\xi)\right]^{-1} \cdot
$$

Finally, we shall prove that we can choose suitable measures $m_{N}$ on $R^{\prime N}$ such that $\int_{\xi_{1} \geqq 1} d m_{N}(\xi)$ is independent from the dimension $N$.

Put

$$
d m_{N}(\xi)=(2 \pi)^{-N / 2} \exp \left(-\frac{|\xi|^{2}}{2}\right) d \xi
$$

then this measure $m_{N}$ fulfills the conditions i) iii) of this section, and moreover it is the product of one-dimensional Gaussian measures. Thus,

$$
\int_{\xi_{1} \geqq 1} d m_{N}(\xi)=\frac{1}{\sqrt{2 \pi}} \int_{1}^{\infty} \exp \left(-\frac{\xi_{1}^{2}}{2}\right) d \xi_{1} .
$$

So, we have proved the condition b) in the fundamental lemma;

$$
1-\mu(S) \leqq \gamma_{0}(\varepsilon+D)
$$

where

$$
\left.\gamma_{0}=\sqrt{2 \pi}\left[\int_{1}^{\infty} \exp \left(-\frac{\xi_{1}^{2}}{2}\right) d \xi_{1}\right]^{-1} \doteqdot 6.4 \text {. (q. e. d. }\right)
$$

\section{§14. Kolmogorov's theorem}

We can get Kolmogorov's theorem as a corollary of Minlos' theorem, as shown below.

Let $L$ be the direct sum of real lines, i.e.

$$
L=\left\{\xi=\left(\xi_{1}, \xi_{2}, \cdots\right) \mid \xi_{i} \in R^{1},{ }^{\exists} n ; i \geqq n \Rightarrow \xi_{i}=0\right\} \text { 。 }
$$

It is the union of $R^{n}=\left\{\left(\xi_{1}, \xi_{2}, \cdots, \xi_{n}, 0,0, \cdots\right)\right\}$. Defining a topology on $L$ by the family of semi-norms $\|\xi\|_{a}=\operatorname{Max}_{i}\left(\left|\xi_{i}\right| / a_{i}\right)$, where $a=\left(a_{1}, a_{2}, \cdots\right)$ is any positive sequence, $L$ becomes a nuclear space.

Suppose that $\chi(\xi)$ is a positive definite function on $L$. Since the matrix;

$$
\left(\begin{array}{lll}
\chi(0) & \chi(\xi) & \chi(\xi+\eta) \\
\overline{\chi(\xi)} & \chi(0) & \chi(\eta) \\
\overline{\chi(\xi+\eta)} & \overline{\chi(\eta)} & \chi(0)
\end{array}\right)
$$


is positive definite, we have $|\chi(\xi+\eta)-\chi(\eta)| \leqq \sqrt{2 \chi(0)} \sqrt{|\chi(\xi)-\chi(0)|}$. Using this inequality, we can show that if $\chi(\xi)$ is continuous on each $R^{n}$, it is continuous necessarily in the topology of $L$.

The dual space of $L$ is the direct product of real lines, i.e.

$$
L^{*}=\left\{x=\left(x_{1}, x_{2}, \cdots\right) \mid x_{i} \in R^{1}\right\} .
$$

Then, $R^{n} \simeq R^{n *}$ can be regarded as a factor space of $L^{*}$. Let $\mathfrak{B}_{n}$ be the whole of the inverse images of Borel sets in $R^{n}$. Then, from Minlos' theorem we get;

Theorem (Kolmogorov)

In case of the direct product of real lines $R^{\infty}=\prod_{k=1}^{\infty} R_{k}^{1}$, any finitely additive probability measure $\mu$ which is completely additive on each $\mathfrak{B}_{n}$, can be extended to a completely additive measure on $\mathfrak{B}_{\infty}$, the smallest $\sigma$-ring which includes all $\mathfrak{B}_{n}$.

\section{Chapter III. Gaussian measures}

Contents : (1) Definition of Gaussian measures on the dual space $L^{*}$ of a nuclear space $L$.

(2) Proof of their $L$-ergodicity, $O$-erodicity and $O$-invariance.

(3) Characterization of an $O$-invariant measure as a superposition of Gaussian measures.

\section{$\S 15$. Characteristic function on a factor space}

Proposition 15.1. Let $L$ be a nuclear space, and $M$ be a closed subspace, then the factor space $L / M$ is also nuclear.

Proof. Let $\varphi$ be the natural homomorphism from $L$ onto $L / M$. For any neighbourhood $\bar{U}$ of 0 in $L / M$, its inverse image $\varphi^{-1}(\bar{U})$ is a neighbourhood of 0 in $L$. Since $L$ is nuclear, there exists a neighbourhood $V$ of 0 such that:

$$
\begin{gathered}
{ }^{\vee} n,{ }^{g} E_{n} \quad(n \text {-dimensional subspace of } L) \\
V \subset E_{n}+\frac{1}{n} \varphi^{-1}(\bar{U}) .
\end{gathered}
$$


Operating $\varphi$ on both hand side, we get $\varphi(V) \subset \varphi\left(E_{n}\right)+\frac{1}{n} \bar{U}$.

Evidently, $\varphi(V) \equiv \bar{V}$ is a neighbourhood of 0 in $L / M$, and the dimension of $\varphi\left(E_{n}\right)$ is at most $n$. (q.e.d.)

Proposition 15.2. Let $\chi(\xi)$ be a positive definite and continuous function on a nuclear space L. Suppose that for a closed subspace $M$ of $L, \chi(\xi)$ is $M$-invariant, i. e.

$$
\checkmark \xi \in L, \quad{ }^{\prime} \xi^{\prime} \in M ; \chi\left(\xi+\xi^{\prime}\right)=\chi(\xi) .
$$

Then, the corresponding measure $\mu$ on $L^{*}$ can be identified with a measure on $M^{\perp}\left(\subset L^{*}\right)$.

Proof. Since $\chi(\xi)$ can be regarded as a positive definite and continuous function on $L / M$, Minlos' theorem enables us to construct the corresponding measure $\mu^{\prime}$ on $(L / M)^{*} \simeq M^{\perp}$ as well as $\mu$ on $L^{*}$. Then, the two triplets $\left\{L^{*}, \mathfrak{B}_{L}, \mu\right\}$ and $\left\{M^{\perp}, \mathfrak{B}_{L} \cap M^{\perp}, \mu^{\prime}\right\}$ are equivalent in the following sense;

$$
\mu(E)=\mu^{\prime}\left(E \cap M^{\perp}\right) \quad \text { for } \quad{ }^{\forall} E \in \mathfrak{B}_{L} \text {. (q. e. d.) }
$$

Now, let $\|\cdot\|_{H}$ be a continuous Hilbertian semi-norm on a nuclear space $L$, and suppose that $\chi(\xi)$ is positive definite and continuous with respect to the semi-norm $\|\cdot\|_{H}$. Then, putting $M=\left\{\xi \in L \mid\|\xi\|_{H}=0\right\}, \chi(\xi)$ is $M$-invariant, so that the corresponding measure $\mu$ can be regarded as a measure on $M^{\perp}$.

On the other hand, the semi-norm $\mid i \cdot \|_{H}$ becomes a Hilbertian norm on $L / M$. Let $\mathfrak{S}$ be the completion of $L / M$ in the norm $\|\cdot\|_{H}$. Since the factor space $L / M$ (with the factor topology of $L$ ) can be imbedded continuously into $\mathfrak{E}$, we get $\mathfrak{S}^{*} \subseteq(L / M)^{*} \simeq M^{\perp}$. Identifying $\mathfrak{S}$ with $\mathfrak{S}^{*}$, we can regard as $L / M \cong M^{\perp}$.

Proposition 15. 3. Let $L$ be complete and nuclear, and $M$ be a subspace mentioned above. By the natural imbedding, $L / M$ is mapped into $M^{\perp}$ densely

Proof. Denoting the isomorphism of $\mathfrak{S C}_{\mathrm{S}}$ onto $\mathfrak{S}^{*}$ with $\psi$, it is sufficient to show that if a linear continuous function on $L^{*}$ vanishes on $\psi(L / M)$, it must vanish also on $M^{\perp}$. 
From Mackey-Arens' theorem [11], if $L$ is complete and nuclear, we have $L^{* *}=L$. Hence, we should only show that for $\xi \in L$,

$$
v_{\bar{\eta}} \in L / M, \xi(\psi(\bar{\eta}))=0 \Rightarrow{ }^{v} x \in M^{\perp}, \xi(x)=0 .
$$

However, from the definition of $\psi$, we have $\xi(\psi(\bar{\eta}))=\langle\bar{\eta}, \bar{\xi}\rangle_{H}$. So that, $\xi(\psi(\bar{\eta}))=0$ for ${ }^{\nabla} \bar{\eta} \in L / M$ implies that $\|\xi\|_{H}=0$, hence $\xi \in M$. Thus, for any $x \in M^{\perp}, \xi(x)=0$.

Example. In case that $L=(\varphi)$, letting $\|\cdot\|_{H}$ be the ordinary $L^{2}-$ norm, evidently $M=\{0\}$. Thus, by the natural imbedding, $(\varphi)$ is mapped into $\left(\varphi^{\prime}\right)$ densely.

From the next section on, in order to avoid the complicated situations, we shall assume that $\|\cdot\|_{H}$ is a continuous Hilbertian norm on a nuclear space $L$. Even for the case where $\|\cdot\|_{H}$ is a semi-norm, the results in the following sections are kept valid, replacing $L$ and $L^{*}$ by $L / M$ and $(L / M)^{*} \simeq M^{\perp}$, respectively.

\section{§16. $L$-quasi-invariance and $\mathcal{O}$-quasi-invariance}

Let $\|\cdot\|_{H}$ be a continuous Hilbertian norm on a complete nuclear space $L$. As seen in the previous section, we can imbed $L$ into $L^{*}$ densely, i.e. $L \subset \mathfrak{S} \simeq \mathfrak{S}^{*} \subset L^{*}$. Then, for any $\xi \in L$, we can define the translation $t_{\xi}$ on $L^{*}$ as follows ; $t_{\xi}: x \in L^{*} \rightarrow x+\xi \in L^{*}$.

Whole of these translations $\left\{t_{\xi}\right\}$ forms a group, which we shall identify with $L$. Then, by Definitions 1.2 and 1.3 of $\S 1$, we can define the concept of $L$-invariance, $L$-quasi-invariance, and $L$ ergodicity for measures on $L^{*}$.

As we have proved in Chapter I, any Borel measure on $L^{*}$ is not $L^{*}$-quasi-inv. Therefore, we shall content ourselves with $L$ quasi-invariant measures, and later as an example of such measures, we shall consider about Gaussian measures.

For any $x \in L^{*}$, we define the translated measure $\tau_{x} \mu$ of the given measure $\mu$. Then;

1) $L$-quasi-invariance of $\mu$ implies that of $\tau_{x} \mu$,

2) $L$-ergodicity of $\mu$ implies that of $\tau_{x} \mu$.

On the other hand, non-existence of $L^{*}$-quasi-inv. measure shows us that 


$$
{ }^{3} x \in L^{*} ; \mu \nsim \tau_{x} \mu \text {. }
$$

Thus, if an $L$-quasi-inv. (or $L$-ergodic) measure $\mu$ is given, we can construct another $L$-quasi-inv. (or $L$-ergcdic) measure $\tau_{x} \mu$ by a suitable translation.

Definition 16.1. Let $\|\cdot\|_{H}$ be a continuous Hilbertian norm on a nuclear space $L$, and let $\mathfrak{S}$ be the completion of $L$ with respect to the norm $\|\cdot\|_{H}$.

An unitary operator $u$ on $\mathfrak{S}$ is called a rotation of $L$, if it satisfies;

1) $u$ maps $L$ onto $L$,

2) $u$ is homeomorphic on $L$.

Whole of rotations of $L$ forms a group, which we call the rotation group of $L$ and denote it with $O_{H}(L)$.

For any $u \in O_{H}(L)$, its adjoint operator $u^{*}$ becomes a homeomorphic transformation on $L^{*}$. Thus, identifying $u^{*}$ with $u^{-1}$, (i.e. identifying $u$ with $\left.u^{-1 *}\right), O_{H}(L)$ can be regarded as a transformation group on $L^{*}$.

Then, by Definitions 1.2 and 1.3 of $\S 1$, the concept of $O_{H}(L)$ invariance, etc. can be defined for measures on $L^{*}$. But, for simplicity of notations, unless any confusion is expected, we shall write $O$ instead of $O_{H}(L)$. Hence, we shall write $O$-inv., $O$-quasi-inv., or $O$-ergodic instead of $O_{H}(L)$-invariant, etc.

Proposition 16.1. Let $\mu$ be a finite measure on the dual space $L^{*}$ of a nuclear space $L . \quad \mu$ is $O$-invariant if and only if the corresponding characteristic function $\chi(\xi)$ depends only on $\|\xi\|_{H}$.

Proof. Sufficiency: Suppose that $\chi(\xi)$ is a function of $\|\xi\|_{H}$ and put $\chi(\xi)=\varphi\left(\|\xi\|_{H}^{2}\right)$. For any $u \in O_{H}(L)$, let $\chi_{u}(\xi)$ be the characteristic function of $\tau_{u} \mu$, then

$$
\begin{aligned}
\chi_{u}(\xi) & =\int \exp [i \xi(x)] d\left(\tau_{u} \mu\right)(x)=\int \exp \left[i \xi\left(u^{-1} x\right)\right] d \mu(x) \\
& =\int \exp [i u \xi(x)] d \mu(x)=\chi(u \xi) \\
& =\varphi\left(\|u \xi\|_{H}^{2}\right)=\varphi\left(\|\xi\|_{H}^{2}\right)=\chi(\xi) .
\end{aligned}
$$

Since a measure on $L^{*}$ is determined uniquely from the character- 
istic function on $L$, this equality implies that $\tau_{u} \mu=\mu$.

Necessity: Suppose that $\mu$ is $O$-invariant. For any $u \in O_{H}(L)$, we have $\chi(u \xi)=\chi_{u}(\xi)=\chi(\xi)$. So that, it is sufficient to show that;

$$
\|\xi\|_{H}=\|\eta\|_{H} \Rightarrow{ }^{\exists} u \in O_{H}(L) ; \eta=u \xi .
$$

For any $\xi, \eta \in L$, let $R$ be the two-dimensional subspace of $L$ which is generated from $\{\xi, \eta\}$. If $\|\xi\|_{H}=\|\eta\|_{H}, \xi$ is mapped to $\eta$ by a suitable rotation $u_{R}$ of $R$. However, since $R$ is finite dimensional, $u_{R}$ can be extended to a rotation $u$ of $L$, i. e. to an unitary operator on $\mathfrak{S}$ which is homeomorphic on $L$. Thus, ${ }^{3} u \in O_{H}(L)$; $u \xi=\eta$. (q. e. d.)

Note: The way how to extend $u_{R}$ to $u$;

Decompose $\mathfrak{S}$ orthogonally into the sum of $R$ and $R^{\perp}$ (=orthogonal complement of $R$ in $\mathfrak{S}$ ), then putting

$$
u= \begin{cases}u_{R} & \text { on } R \\ \text { identity } & \text { on } R^{\perp},\end{cases}
$$

$u$ becomes a rotation of $L$.

Finally, we remark that if $L$ is separable, Corollary of Prop. 3. $1^{\prime}$ assures the equivalence of four statements in Prop. 1.1. Therefore, we can define $L$-ergodicity (or $O$-ergodicity) by any of these four statements.

\section{$\S 17$. Definition of Gaussian measures}

Definition 17.1. Let $\|\cdot\|_{H}$ be a continuous Hilbertian norm on a nuclear space $L$. It is easily seen that the following $\chi(\xi)$ is continuous and positive definite on L.

$$
\chi(\xi)=\exp \left(-\frac{c^{2}\|\xi\|_{H}^{2}}{2}\right) \quad(c>0)
$$

The corresponding measure on $L^{*}$ is called a Gaussian measure with variance $c^{2}$. (From now on, we denote it with $\mu_{c}$ ).

Proposition 17.1. Let $R_{n}$ be an $n$-dimensional subspace of $L$, and $\left\{\xi_{1}, \xi_{2}, \cdots, \xi_{n}\right\}$ be a complete orthonormal system of $R_{n}\left(\right.$ in $\left.\|\cdot\|_{H}\right)$. Then, for $x \in L^{*}$, putting $\xi_{k}(x)=\bar{x}^{(k)}$, we have the following expression of $\mu_{c, R_{n}} \equiv \mu_{c} \mid \overline{\mathfrak{B}}_{R_{n}}$. 


$$
d_{\mu_{c, R_{n}}}(x)=\left(\frac{1}{\sqrt{2 \pi} c}\right)^{n} \exp \left[-\frac{1}{2 c^{2}} \sum_{k=1}^{n} \bar{x}^{(k) 2}\right] d \bar{x}^{(1)} d \bar{x}^{(2)} \ldots d \bar{x}^{(n)} .
$$

(This expression depends only on the dimension $n$ of $R_{n}$, namely it is same for all $n$-dimensional subspace $R_{n}$ and for all choices of the base $\left\{\xi_{k}\right\}$.)

Proof. For $\xi \in R_{n}$, writing it as $\xi=\sum_{k=1}^{n} a_{k} \xi_{k}$ ( $a_{k}$ : real), we have

$$
\begin{aligned}
& \chi(\xi)=\exp \left(-\frac{c^{2}}{2} \sum_{k=1}^{n} a_{k}^{2}\right), \quad \text { hence we have : } \\
& \chi(\xi)=\left(\frac{1}{\sqrt{2 \pi} c}\right)^{n} \int \exp \left[i \sum_{k=1}^{n} a_{k} \bar{x}^{(k)}-\frac{1}{2 c^{2}} \sum_{k=1}^{n} \bar{x}^{(k) 2}\right] d^{n} \bar{x} .
\end{aligned}
$$

(Here, $d^{n} \bar{x}$ means $d \bar{x}^{(1)} d \bar{x}^{(2)} \ldots d \bar{x}^{(n)}$ )

However, since $\sum_{k=1}^{n} a_{k} \bar{x}^{(k)}=\xi(x)$, we have the expression (2) from the one-to-one correspondence between characteristic functions and measures. (q. e.d.)

(Note): Since $L$ is nuclear, it is separable with respect to the norm $\|\cdot\|_{H}$. (c.f. Prop. 11.3). So that the expression (2) allows us to write formally;

$$
d \mu_{c}(x)=\lim _{n \rightarrow \infty}\left[\left(\frac{1}{\sqrt{2 \pi} c}\right)^{n} \exp \left(-\frac{1}{2 c^{2}} \sum_{k=1}^{n} \bar{x}^{(k) 2}\right) d \bar{x}^{(1)} d \bar{x}^{(2)} \ldots d \bar{x}^{(n)}\right]
$$

(Here, consider a complete orthonormal system $\left\{\xi_{k}\right\}$ of $L$ in $\|\cdot\|_{H}$ and put $\left.\bar{x}^{(k)}=\xi_{k}(x)\right)$.

In this sense, $\mu_{c}$ is the limit measure of finite dimensional Gaussian measures (letting the dimension $n \rightarrow \infty$ ).

\section{$\S 18$. Proof of L-ergodicity}

Proposition 18.1. Gaussian measure $\mu_{c}$ is L-quasi-invariant.

Proof. It is sufficient to show that;

$$
v \xi \in L ; \tau_{\xi} \mu_{c} \sim \mu_{c} .
$$

Let $\chi_{\xi}(\eta)(\eta \in L)$ be the characteristic function of $\tau_{\xi} \mu_{c}$, namely;

$$
\chi_{\xi}(\eta)=\int \exp [i \eta(x)] d\left(\tau_{\xi} \mu_{c}\right)(x) .
$$


Let $R_{2}$ be the two-dimensional subspace of $L$ which is generated from $\{\xi, \eta\}$ and let $\left\{\xi_{1}, \xi_{2}\right\}$ be a complete orthonormal system (in $\left.\|\cdot\|_{H}\right)$ of $R_{2}$. Then

$$
\chi_{\xi}(\eta)=\int \exp [i \eta(x)] \frac{d\left(\tau_{\xi} \mu_{c, R_{2}}\right)}{d \mu_{c, R_{2}}}(x) d \mu_{c, R_{2}}(x) .
$$

However, from (2) we have

$$
\begin{aligned}
\frac{d\left(\tau_{\xi} \mu_{c, R_{2}}\right)}{d \mu_{c, R_{2}}}(x) & =\exp \left[-\frac{1}{2 c^{2}} \sum_{k=1}^{2}\left\{\left(\bar{x}^{(k)}+\bar{\xi}^{(k)}\right)^{2}-\bar{x}^{(k) 2}\right\}\right] \\
& =\exp \left[-\frac{1}{2 c^{2}} \sum_{k=1}^{2}\left(2 \bar{x}^{(k)} \xi^{(k)}+\bar{\xi}^{(k) 2}\right)\right] .
\end{aligned}
$$

Remember that $\xi^{(k)}=\xi_{k}(\xi)=\left\langle\xi, \xi_{k}\right\rangle_{H}$, then it is evident that $\xi=\xi^{(1)} \xi_{1}$ $+\bar{\xi}^{(2)} \xi_{2}$ 。

Hence, $\sum_{k=1}^{2} \bar{x}^{(k)} \bar{\xi}^{(k)}=\sum_{k=1}^{2} \bar{\xi}^{(k)} \xi_{k}(x)=\xi(x)$, and $\sum_{k=1}^{2} \bar{\xi}^{(k) 2}=\|\xi\|_{H}^{2}$.

Substituting these into (5), we get

$$
\frac{d\left(\tau_{\xi} \mu_{c, R_{2}}\right)}{d \mu_{c, R_{2}}}(x)=\exp \left[-\frac{1}{2 c^{2}}\left(2 \xi(x)+\|\xi\|_{H}^{2}\right)\right] .
$$

This does not depend on $\eta$ any more. Substituting it into (4), we have ;

$$
\chi_{\xi}(\eta)=\int \exp [i \eta(x)] \exp \left[-\frac{1}{2 c^{2}}\left(2 \xi(x)+\|\xi\|_{H}^{2}\right)\right] d \mu_{c}(x) . \quad\left(\text { for } v_{\eta \in L}\right)
$$

Since the characteristic function determines the corresponding measure uniquely, we have;

$$
\frac{d\left(\tau_{\xi} \mu_{c}\right)}{d \mu_{c}}(x)=\exp \left[-\frac{1}{2 c^{2}}\left(2 \xi(x)+\|\xi\|_{H}^{2}\right)\right] .
$$

From this, it is evident that:

$$
\tau_{\xi} \mu_{c} \lesssim \mu_{c} .
$$

Since (7) holds for any $\xi \in L$, we have also ;

$$
\tau_{-\xi} \mu_{c} \lesssim \mu_{c} \text { hence } \mu_{c} \lesssim \tau_{\xi} \mu_{c} .
$$

Comparing (7) with $\left(7^{\prime}\right)$, we have obtained;

$$
\tau_{\xi} \mu_{c} \sim \mu_{c} .
$$


Proposition 18.2. For Gaussian measure $\mu_{c}$, the four statements in Prop. 1.1 are equivalent with each other, so that we can use any of them as the definition of ergodicity.

Proof. In case that $L$ is weakly separable, we have already proved this prop. in Corollary of Prop. 3. $1^{\prime}$.

However, since the characteristic function of Gaussian measure is continuous with respect to $\|\cdot\|_{H}$, we can prove in a similar way as in Prop. 3. 1, that the separability of $L$ with respect to any Hilbertian norm is sufficient for the equivalence of the four statements.

On the other hand, a nuclear space $L$ is always separable in this meaning (Prop. 11.3). (q.e.d.)

Proposition 18. 3. Gaussian measure $\mu_{c}$ is L-ergodic.

Proof. We shall check the statement (3) in Prop. 1. 1. Namely, we shall show that for any $\mathfrak{B}_{L}$-measurable function $\varphi(x)$;

$\forall \xi \in L ; \varphi(x)=\varphi(x+\xi) \Rightarrow \varphi(x)=$ constant for $\mu_{c}$-almost all $x$.

Without loss of generality, we can assume that $\varphi(x)$ is bounded.

Since $L$ is separable with respect to any continuous Hilbertian norm, we can show in a similar way as in Prop. 3.1', that;

$$
\begin{gathered}
{ }^{g} R_{n} \text { : finite dimenssional subspace of } L ; \\
\cup L_{\mu_{c}, n}^{2}\left(L^{*}\right) \text { is dense in } L_{\mu_{c}}^{2}\left(L^{*}\right) .
\end{gathered}
$$

(Here, $\mu_{c, n} \equiv \mu_{c} \mid \mathfrak{B}_{R_{n}}$. We assume that $R_{1} \subseteq R_{2} \subseteq R_{3} \subseteq \cdots$ ).

For simplicity, we shall write $M_{n}$ instead of $L_{\mu_{c}, n}^{2}\left(L^{*}\right)$. Decompose $\varphi(x) \in L_{\mu_{c}}^{2}\left(L^{*}\right)$ orthogonally into the form ;

$$
\varphi(x)=\varphi_{n}^{(1)}(x)+\varphi_{n}^{(2)}(x)
$$

where $\varphi_{n}^{(1)}(x) \in M_{n}$, and $\varphi_{n}^{(2)}(x) \in M_{n}^{\perp}$ (=orthogonal complement of $M_{n}$ in $\left.L_{\mu_{c}}^{2}\left(L^{*}\right)\right)$. The sequence $\left\{M_{n}\right\}$ is monotonic increasing as well as $\left\{R_{n}\right\}$, hence;

$$
\varphi(x)=\lim _{n} \varphi_{n}^{(1)}(x) \quad \text { in } \quad L^{2} \mu_{c}\left(L^{*}\right) .
$$

On the other hand, from (8) we get;

$$
\varphi(x+\xi)=\varphi_{n}^{(1)}(x+\xi)+\varphi_{n}^{(2)}(x+\xi) .
$$


It is easily seen that if $\varphi(x)$ is bounded, then $\varphi_{n}^{(1)}(x)$ and $\varphi_{n}^{(2)}(x)$ are also bounded, and for any $\xi \in L, \varphi_{n}^{(1)}(x+\xi) \in M_{n}$.

Now, if we could find the relation;

$$
{ }^{\forall} \xi \in R_{n} ; \varphi_{n}^{(2)}(x+\xi) \in M_{n}^{\perp},
$$

we would know, from the uniqueness of orthogonal decomposition, that the condition $\forall \xi \in L ; \varphi(x)=\varphi(x+\xi)$ implies :

$$
\forall \xi \in R_{n} ; \varphi_{i 2}^{(1)}(x)=\varphi_{n}^{(1)}(x+\xi) .
$$

However, $\varphi_{n}^{(1)}(x)$ can be regarded as a function on the factor space $L^{*} / R_{n}^{\perp}$, which is isomorphic with $R_{n}^{*}$ and hence (imbedding $R_{n}$ into $L^{*}$ ) with $R_{n}$. So that,

$$
\varphi_{n}^{(1)}(x+\xi)=\varphi_{n}^{(1)}(x) \quad \text { for } \quad \forall \xi \in R_{n}
$$

implies that $\varphi_{n}^{(1)}(x)=$ constant for $\mu_{c}$-almost all $x$.

Then, from (9) we see that $\varphi(x)$ is the limit function of constant functions, thus $\varphi(x)$ itself must be constant. Therefore, the proposition has been proved except the relation (10).

Proof of (10). It is sufficient to show;

$$
\begin{gathered}
{ }^{\vee} \psi(x) \in M_{n} \text { and bounded, }{ }^{v} \xi \in R_{n} ; \\
\left\langle\phi_{n}^{(2)}(x+\xi), \quad \psi(x)\right\rangle_{\mu_{c}}=0 .
\end{gathered}
$$

However,

$$
\int \phi_{n}^{(2)}(x+\xi) \overline{\psi(x)} d \mu_{c}(x)=\int \phi_{n}^{(2)}(x) \overline{\psi(x-\xi)} \frac{d\left(\tau_{-\xi} \mu_{c}\right)}{d \mu_{c}}(x) d \mu_{c}(x),
$$

and from (6) we know that;

$$
\frac{d\left(\tau_{-\xi} \mu_{c}\right)}{d \mu_{c}}(x)=\exp \left[\frac{1}{2 c^{2}}\left(2 \xi(x)-\|\xi\|_{H}^{2}\right)\right],
$$

so that for any $\xi \in R_{n}$,

$$
\psi(x-\xi) \frac{d\left(\tau_{-\xi} \mu_{c}\right)}{d \mu_{c}}(x) \in M_{n} .
$$

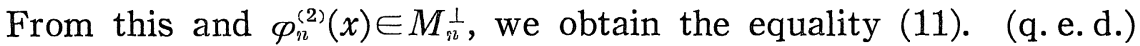

(Note): As seen in this proof, for $L$-ergodicity of a measure $\mu$ it is essentially important that 


$$
\xi \in R_{n} \Rightarrow \frac{d\left(\tau_{\xi} \mu\right)}{d \mu}(x) \in M_{n}
$$

\section{$\S 19 . \quad$ Proof of 0 -ergodicity}

Proposition 19.1. Gaussian measure $\mu_{c}$ is O-invariant.

Proof. Prop. 16. 1 assures the $O$-invariance of Gaussian measure, because the characteristic function depends only on $\|\xi\|_{H}$ as given in (1) of $\S 17$.

Proposition 19.2. Gaussian measure $\mu_{c}$ is O-ergodic.

Proof. As seen in Prop. 18. 2 the four statements in Prop. 1. 1 are equivalent with each other, so that we shall check the statement (3) in Prop. 1.1. Namely, we shall show that for any bounded $\mathfrak{B}_{L}$-measurable function $\varphi(x)$ on $L^{*}$;

$$
{ }^{\forall} u \in O_{H}(L) ; \varphi(x)=\varphi(u x) \Rightarrow \varphi(x)=\text { constant for } \mu_{c} \text {-almost all } x \text {. }
$$

We shall follow just the same way in the proof of Prop. 18.3, until we reach the equalities (8) and (9). Instead of $\left(8^{\prime}\right)$, we get

$$
\varphi(u x)=\varphi_{n}^{(1)}(u x)+\varphi_{n}^{(2)}(u x)
$$

but in general $\varphi_{n}^{(1)}(u x) \notin M_{n}$.

Now, suppose that $\varphi(x)=\varphi(u x)$ in $L_{\mu_{c}}^{2}\left(L^{*}\right)$, then

$$
\varphi_{n}^{(1)}(x)+\varphi_{n}^{(2)}(x)=\varphi_{n}^{(1)}(u x)+\varphi_{n}^{(2)}(u x),
$$

thus we have;

$$
\left\|\varphi_{n}^{(1)}(u x)-\varphi_{n}^{(1)}(x)\right\|_{\mu_{c}}=\left\|\varphi_{n b}^{(2)}(x)-\varphi_{n}^{(2)}(u x)\right\|_{\mu_{c}} \leqq 2\left\|\varphi_{n}^{(2)}(x)\right\|_{\mu_{c}},
$$

where the last irequality comes from the $O$-invariance of the measure $\mu_{c}$ 。

Remembering the note after the proof of Prop. 16.1, we can easily show that;

$$
\left.{ }^{\sharp} u \in O_{H}(L) ; R_{n} \perp u^{-1} R_{n} \quad \text { (in } \mathfrak{S}\right) .
$$

(We extend a rotation of a $2 n$-dimensional subspace $R_{2 n}$ which contains $R_{n}$, to a rotation of $L$ ). For this $u$, we shall calculate the left hand side of (12). Then, 


$$
\begin{aligned}
& \left\|\varphi_{n}^{(1)}(u x)-\varphi_{n}^{(1)}(x)\right\|_{\mu_{c}}^{2}=\int\left|\varphi_{n}^{(1)}(u x)-\varphi_{n}^{(1)}(x)\right|^{2} d \mu_{c}(x) \\
& =2 \int\left|\varphi_{n}^{(1)}(x)\right|^{2} d \mu_{c}(x)-2 \operatorname{Re} \int \varphi_{n}^{(1)}(u x) \overline{\varphi_{n}^{(1)}(x)} d \mu_{c}(x) .
\end{aligned}
$$

From Prop. 17.1, the measure $\mu_{c}, R_{2 n}$ is the direct product of $\mu_{c, R_{n}}$ and $\mu_{c, u^{-1} R_{n}}$, so that;

$$
\begin{aligned}
\int \varphi_{n}^{(1)}(u x) \overline{\varphi_{n}^{(1)}(x)} d_{\mu_{c}}(x) & =\int \varphi_{n}^{(1)}(u x) d_{\mu_{c}}(x) \times \int \overline{\varphi_{n}^{(1)}(x)} d \mu_{c}(x) \\
& =\left|\int \varphi_{n}^{(1)}(x) d \mu_{c}(x)\right|^{2} .
\end{aligned}
$$

Substituting this into (13), we have

$$
\left\|\varphi_{n}^{(1)}(u x)-\varphi_{n}^{(1)}(x)\right\|_{\mu_{c}}^{2}=2\left[\int\left|\varphi_{n 2}^{(1)}(x)\right|^{2} d \mu_{c}(x)-\left|\int \varphi_{n}^{(1)}(x) d \mu_{c}(x)\right|^{2}\right] .
$$

Thus, the inequality (12) is equivalent with;

$$
\int\left|\varphi_{n}^{(1)}(x)\right|^{2} d \mu_{c}(x)-\left|m_{n}\right|^{2} \leqq 2\left\|\varphi_{n}^{(2)}(x)\right\|_{\mu_{c}}^{2},
$$

where

$$
m_{n}=\int \varphi_{n}^{(1)}(x) d \mu_{c}(x)
$$

Letting $n \rightarrow \infty$, since $\lim _{n} \varphi_{n}^{(1)}(x)=\varphi(x)$ and $\lim _{n} \varphi_{n}^{(2)}(x)=0 \quad$ in $L_{\mu_{c}}^{2}\left(L^{*}\right)$,

we have

$$
\begin{gathered}
\int|\varphi(x)|^{2} d \mu_{c}(x)-|m|^{2} \leqq 0, \\
m=\int \varphi(x) d \mu_{c}(x) \\
\int|\varphi(x)-m|^{2} d \mu_{c}(x) \leqq 0,
\end{gathered}
$$

where

Therefore,

but this means that $\varphi(x)=m$ for $\mu_{c}$-almost all $x$. Thus $\varphi(x)$ is a constant function (modulo $\mu_{c}$-null set). (q. e.d.)

\section{$\S 20$. Singularity with each other}

In this section, we shall show that $\mu_{c} \nsim \mu_{c^{\prime}}$ for $c \neq c^{\prime}$. For this purpose, we could use the criterion of Prop. 3.2. But more strongly, we shall construct concretely a set $A$ that fulfills $\mu_{c}(A)=0$ but $\mu_{c^{\prime}}(A)=1$. Such $A$ can be constructed in some different ways. 
Proposition 20.1. Let $\|\cdot\|_{H}$ be a continuous Hilbertian norm on a nuclear space $L$, and $\left\{\xi_{k}\right\}$ be a complete orthonormal system of $L$ in $\|\cdot\|_{H}$.

Define the function $f(x)$ on $L^{*}$ as follows;

$$
f(x)=\varlimsup_{k} \frac{\left|\xi_{k}(x)\right|}{\sqrt{2} \log k} .
$$

Then, putting $A_{c}=f^{-1}(c)=\left\{x \in L^{*} \mid f(x)=c\right\}$, we have $\mu_{c}\left(A_{c}\right)=1$. (Evidently, $A_{c} \cap A_{c^{\prime}}=\phi$ if $c \neq c^{\prime}$ ).

(Note): It is easily seen that $f(x)$ is $\mathfrak{B}_{L}$-measurable and that for ${ }^{v} x \in L^{*}$ and ${ }^{\forall} \xi \in L ; f(x+\xi)=f(x)$. Since $\mu_{c}$ is $L$-ergodic, this shows that $f(x)$ is constant for $\mu_{c}$-almost all $x$. We must prove that this constant is just $c$.

Proof. For $k \geqq 2$ and $\alpha>0$, put;

$$
E_{k}^{\alpha}=\left\{x \in L^{*} \mid \frac{\left|\xi_{k}(x)\right|}{\sqrt{2} \frac{\overline{l o g} k}{\log } \geqq \alpha}\right\}
$$

From the equality (2) of $\S 17$, we have

$$
\mu_{c}\left(E_{k}^{\alpha}\right)=2 \frac{1}{\sqrt{2 \pi} c} \int_{\alpha \sqrt{2 \log k}}^{\infty} \exp \left[-\frac{\bar{x}^{(k) 2}}{2 c^{2}}\right] d \bar{x}^{(k)} .
$$

We shall estimate this integral. It is easily seen that for ${ }^{v} c^{\prime}<c$

$$
\begin{gathered}
{ }^{\exists} \beta>0,{ }^{V} \bar{x}^{(k)} \geqq \alpha \sqrt{2 \log k} ; \\
\beta \bar{x}^{(k)} \exp \left[-\frac{\bar{x}^{(k) 2}}{2 c^{\prime 2}}\right] \leqq \exp \left[-\frac{\bar{x}^{(k) 2}}{2 c^{2}}\right] \leqq \frac{\bar{x}^{(k)}}{\alpha \sqrt{2} \log \bar{k}} \exp \left[-\frac{\bar{x}^{(k) 2}}{2 c^{2}}\right]
\end{gathered}
$$

so that integrating each of them, we get;

$$
\beta \sqrt{\frac{2}{\pi}} \frac{c^{\prime 2}}{c} k^{-\alpha^{2} / c^{\prime 2}} \leqq \mu_{c}\left(E_{k}^{\alpha}\right) \leqq \sqrt{\frac{2}{\pi}} \frac{c}{\alpha \sqrt{ } 2 \log k} k^{-\alpha^{2} / c^{2}} .
$$

Thus, we have the following relations;

$$
\begin{aligned}
& \alpha>c \Rightarrow \sum_{k=2}^{\infty} \mu_{c}\left(E_{k}^{a}\right)<\infty \\
& \alpha<c \Rightarrow \sum_{k=2}^{\infty} \mu_{c}\left(E_{k}^{a}\right)=\infty .
\end{aligned}
$$


In case of $(16)$, we know $\mu_{c}\left(\varlimsup_{k} E_{k}^{\alpha}\right)=0$. Since $f(x)>\alpha$ implies $x \in \varlimsup_{k} E_{k}^{\alpha}$, we have $\mu_{c}(\{x \mid f(x)>\alpha\})=0$, thus letting $\alpha \rightarrow c+0$,

$$
\mu_{c}(\{x \mid f(x)>c\})=0 .
$$

On the other hand, in case of $\left(16^{\prime}\right)$, putting ;

$$
B_{n}^{\alpha}=\left\{x \in L^{*} \mid{ }^{\vee} k \geqq n,-\frac{\left|\xi_{k}(x)\right|}{\sqrt{2 \log k}}<\alpha\right\},
$$

we see that

$$
\mu_{c}\left(B_{n}^{\alpha}\right)=\prod_{k=n}^{\infty}\left(1-\mu_{c}\left(E_{k}^{\alpha}\right)\right)=0
$$

Since $f(x)<\alpha$ implies $x \in B_{n}^{\alpha}$ for some $n$, we have $\mu_{c}(\{x \mid f(x)<\alpha\})$ $=0$, thus letting $\alpha \rightarrow c-0$,

$$
\mu_{c}(\{x \mid f(x)<c\})=0 .
$$

From (17) and $\left(17^{\prime}\right)$, it is evident that $\mu_{c}(\{x \mid f(x)=c\})=1$, i. e. $f(x)=c$ for $\mu_{c}$-almost all $x$. (q.e. d.)

For another example, the following set $A_{c}$ also fulfills $\mu_{c}\left(A_{c}\right)=1$.

Proposition 20.2. Let $\left\{\xi_{k}\right\}$ be of the same meaning in the previous proposition, then we have;

$$
\lim _{n \rightarrow \infty}\left(\frac{1}{n} \sum_{k=1}^{n}\left(\xi_{k}(x)\right)^{2}\right)=c^{2} \quad \text { for } \mu_{c} \text {-almost all } x \text {. }
$$

(Namely, putting

$$
A_{c}=\left\{x \in L^{*} \mid \lim _{n \rightarrow \infty}\left(\frac{1}{n} \sum_{k=1}^{n}\left(\xi_{k}(x)\right)^{2}\right)=c^{2}\right\},
$$

we have $\mu_{c}\left(A_{c}\right)=1$. Evidently, $A_{c} \cap A_{c^{\prime}}=\phi$ if $c \neq c^{\prime}$.)

Proof. (Omitted). It can be proved by Kolmogorov's strong law of large numbers.

\section{$\S 21$. Characterization of $O$-invariant measures}

Two fundamental questions about ergodic decomposition of measures are: (1) to find concretely all ergodic measures, and (2) to decompose any given quasi-invariant measure into a sum of 
ergodic measures. Though in general they are difficult to answer, in case of $O$-invariant measures the affirmative answers can be get rather easily.

Proposition 21. 1. Let $\|\cdot\|_{H}$ be a continuous Hilbertian norm on a nuclear space $L$. On the dual space $L^{*}$ any $O$-invariant measure (except the Dirac measure) can be expressed as a superposition of Gaussian measures.

Namely, a finite measure $\mu$ on $L^{*}$ is O-invariant if and only if it can be expressed as;

$$
{ }^{\forall} A \in \mathfrak{B}_{L} ; \mu(A)=\int_{(0, \infty)} \mu_{c}(A) d m(c)+\alpha \delta(A) .
$$

where $\alpha \geqq 0$ and $m(c)$ is a finite measure on the half-line $(0, \infty)$.

$\delta(A)$ means the Dirac measure on the origin of $L^{*}$, i.e.

$$
\delta(A)=\left\{\begin{array}{lll}
1 & \text { if } & 0 \in A \\
0 & \text { if } & 0 \notin A .
\end{array}\right.
$$

Proof of sufficiency. Since both $\mu_{c}(A)$ and $\delta(A)$ are $O$-invariant, any measure $\mu$ in the form of (18) is evidently $O$-invariant.

For the proof of necessity, we need some lemmas.

Definition 21. 1. A real function $\varphi(t)$ defined on the closed halfline $[0, \infty)$, is called completely monotonic if it satisfies;

$$
\begin{aligned}
& (-1)^{n} \Delta_{\alpha}^{(n)} \varphi(t) \equiv \sum_{k=0}^{n}(-1)^{k}\left(\begin{array}{l}
n \\
k
\end{array}\right) \varphi(k \alpha+t) \geqq 0 \\
& \quad \text { for } n=0,1,2, \cdots,{ }^{*} t \geqq 0, \quad{ }^{\forall} \alpha \geqq 0 .
\end{aligned}
$$

Lemma (Bernstein's theorem.) Let $\varphi(t)$ be completely monotonic on $[0, \infty)$ and right continuous at $t=0$. Then, there exists a finite measure $\tilde{m}(s)$ on $[0, \infty)$ such that;

$$
\varphi(t)=\int_{(0, \infty)} \exp (-s t) d \tilde{m}(s) \quad \text { for } \quad{ }^{v} t \geqq 0 .
$$

(i. e. $\varphi(t)$ is the Laplace transform of a finite measure.)

For the proof of this Lemma, for instance, c.f. D. V. Widder: "Laplace Transform". 
Now, we shall resume the proof of necessity of Prop. 21.1. Let $\mu$ be an $O$-invariant finite measure on $L^{*}$, then from Prop. 16. 1 the corresponding characteristic function $\chi(\xi)$ depends only on $\|\xi\|_{H}$. Namely, there exists a function $\varphi(t)$ defined on $[0, \infty)$ such that $\chi(\xi)=\varphi\left(\|\xi\|_{H}^{2}\right)$. Since $\chi(\xi)$ is continuous in $\|\cdot\|_{H}, \varphi(t)$ is right continuous at $t=0$. For a while, we assume the complete monotonicity of $\varphi(t)$, which we shall prove later.

Then, from Bernstein's theorem, there exists a finite measure $\tilde{m}(s)$ on $[0, \infty)$ such that $\chi(\xi)=\int_{(0, \infty)} \exp \left(-s\|\xi\|_{H}^{2}\right) d \tilde{m}(s)$. Putting $s=\frac{c^{2}}{2}$, we change the integral variable from $s$ to $c$, then the measure $\tilde{m}(s)$ is transformed to a finite measure $m(c)$ on $[0, \infty)$, and we get;

$$
\chi(\xi)=\int_{(0, \infty)} \exp \left(-\frac{c^{2}}{2}\|\xi\|_{H}^{2}\right) d m(c) .
$$

Remember that $\exp \left(-\frac{c^{2}}{2}\|\xi\|_{H}^{2}\right)$ and 1 (for $v \xi$ ) is the characteristic function of $\mu_{c}$ and $\delta$ respectively. Since the characteristic function determines the measure uniquely, (19) is equivalent with;

$$
{ }^{\mathrm{V}} A \in \mathfrak{B}_{L} ; \mu(A)=\int_{\left(0_{c} \infty\right)} \mu_{c}(A) d m(c)+\alpha \delta(A),
$$

where $\alpha=m(\{0\})$.

Thus, only remained to prove is the fact that $\varphi(t)$ is completely monotonic.

Proposition 21.2. Let $\phi\left(\|\xi\|_{H}^{2}\right)$ be a function on a nuclear space $L$ dependent only on $\|\xi\|_{H}$. If $\varphi\left(\|\xi\|_{H}^{2}\right)$ is positive definite on $L$, then $\varphi(t)$ is necessarily completely monotonic.

We divide the proof into two parts.

Proposition 21.3. Let $\varphi\left(\|\xi\|_{H}^{2}\right)$ be a positive definite function on a nuclear space $L$, then,
a) $\varphi(t) \geqq 0 \quad$ for $\quad \forall t \geqq 0$,
b) $\Delta_{\alpha}^{(1)} \varphi\left(\|\xi\|_{H}^{2}\right) \equiv \varphi\left(\|\xi\|_{H}^{2}+\alpha\right)-\varphi\left(\|\xi\|_{H}^{2}\right)$ is negative definite on $L$. 
Remark. For the validity of Prop. 21.3, it is essential that $L$ is infinite dimensional.

Proof of Prop. 21. 2 using Prop. 21.3. If $\varphi\left(\|\xi\|_{H}^{2}\right)$ is positive definite on $L$, applying Prop. $21.3 \mathrm{~b}$ ), we see that for ${ }^{V} n,{ }^{V} \alpha \geqq 0$; $(-1)^{n} \Delta_{\alpha}^{(n)} \varphi\left(\|\left.\xi\right|_{H} ^{2}\right)$ is a positive definite function on $L$. Then, Prop. 21.3 a) shows us that $(-1)^{n} \Delta_{\alpha}^{(n)} \varphi\left(i \mid \xi \|_{H}^{2}\right)$ is positive for any $\|\xi\|_{H}, \alpha, n$ 。 Thus, $\varphi(t)$ is completely monotonic. (q.e.d.)

Proof of Prop. 21.3. a) For any given $t \geqq 0$ and positive integer $n$, since $L$ is infinite dimensional, there exist $\xi_{1}, \xi_{2}, \cdots, \xi_{n} \in L$ such that;

$$
\left\langle\xi_{i}, \xi_{j}\right\rangle_{H}=t \delta_{i j} \quad(\text { for } \quad 1 \leqq i, j \leqq n) .
$$

If $\varphi\left(\|\xi\|_{H}^{2}\right)$ is positive definite on $L$, then

$$
\begin{aligned}
0 \leqq \sum_{i, j} \varphi\left(\left\|\xi_{i}-\xi_{j}\right\|_{H}^{2}\right) & =n \varphi(0)+\sum_{i \neq j} \varphi\left(\left\|\xi_{i}-\xi_{j}\right\|_{H}^{2}\right) \\
& =n \varphi(0)+n(n-1) \varphi(2 t),
\end{aligned}
$$

so that $\varphi(2 t) \geqq-\frac{1}{n-1} \varphi(0)$.

Letting $n \rightarrow \infty$, we see that $\varphi(2 t) \geqq 0$. Thus, $\varphi(t)$ is a positive function.

b) Putting $\Delta_{a}^{(1)} \varphi\left(\|\xi\|_{H}^{2}\right)=\psi\left(\|\xi\|_{H}^{2}\right)$, we shall show that $\psi\left(\|\xi\|_{H}^{2}\right)$ is negative definite on $L$, namely for any finite set of complex numbers $a_{1}, a_{2}, \cdots, a_{n}$ and for any $\xi_{1}, \xi_{2}, \cdots, \xi_{n} \in L$, we have ;

$$
\sum_{i, j=1}^{n} a_{i} \bar{a}_{j} \psi\left(\left\|\xi_{i}-\xi_{j}\right\|_{H}^{2}\right) \leqq 0
$$

Since $L$ is infinite dimensional, there exists $\xi_{0} \in L$ such that;

$$
\left\langle\xi_{0}, \xi_{i}\right\rangle_{H}=\alpha \delta_{0 i} \quad(\text { for } \quad 0 \leqq i \leqq n)
$$

Using this $\xi_{0}$, we put;

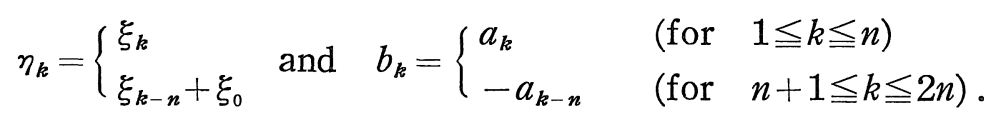

Then, since $\varphi\left(\|\xi\|_{H}^{2}\right)$ is positive definite on $L$, 


$$
\begin{aligned}
0 \leqq & \sum_{i, j=1}^{2 n} b_{i} \bar{b}_{j} \varphi\left(\left\|\eta_{i}-\eta_{j}\right\|_{H}^{2}\right) \\
= & \sum_{i, j=1}^{n} a_{i} \bar{a}_{j} \varphi\left(\left\|\xi_{i}-\xi_{j}\right\|_{H}^{2}\right)-\sum_{i, j=1}^{n} a_{i} \bar{a}_{j} \varphi\left(\left\|\xi_{i}-\xi_{j}-\xi_{0}\right\|_{H}^{2}\right) \\
& -\sum_{i, j=1}^{n} a_{i} \bar{a}_{j} \varphi\left(\left\|\xi_{i}+\xi_{0}-\xi_{j}\right\|_{H}^{2}\right)+\sum_{i, j=1}^{n} a_{i} \bar{a}_{j} \varphi\left(\left\|\xi_{i}-\xi_{j}\right\|_{H}^{2}\right) \\
= & 2\left[\sum_{i, j=1}^{n} a_{i} \bar{a}_{j} \varphi\left(\left\|\xi_{i}-\xi_{j}\right\|_{H}^{2}\right)-\sum_{i, j=1}^{n} a_{i} \bar{a}_{j} \varphi\left(\left\|\xi_{i}-\xi_{j}\right\|_{H}^{2}+\alpha\right)\right] \\
= & 2 \sum_{i, j=1}^{n} a_{i} \bar{a}_{j}\left\{-\psi\left(\left\|\xi_{i}-\xi_{j}\right\|_{H}^{2}\right)\right\} .
\end{aligned}
$$

Thus, we have reached;

$$
\sum_{i, j=1}^{n} a_{i} \bar{a}_{j} \psi\left(\left\|\xi_{i}-\xi_{j}\right\|_{H}^{2}\right) \leqq 0
$$

which shows us that $\psi\left(\|\left.\xi\right|_{H} ^{2}\right)$ is negative definite on $L$. (q.e.d.)

\section{$\S 22 . \quad O$-invariant and ergodic measures}

Proposition 22.1. Let $\|\cdot\|_{H}$ be a continuous Hilbertian norm on a nuclear space $L$, and $\mu$ be an O-invariant measure on $L^{*}$.

a) If $\mu$ is L-quasi-invariant, then

${ }^{\exists} m(c)$ : finite measure on the interval $(0, \infty)$;

$$
{ }^{v} A \in \mathfrak{B}_{L} ; \mu(A)=\int_{(0, \infty)} \mu_{c}(A) d m(c) .
$$

(The Dirac term of (18) drops.)

b) If $\mu$ is L-ergodic, then $\mu=\mu_{c}$ for some $c>0$.

c) If $\mu$ is O-ergodic, then $\mu=\mu_{c}$ for some $c>0$ or $\mu=\delta$.

Proof. a) is evident, for $\delta(A)$ is not $L$-quasi-invariant.

To prove b), we shall show that $m(c)=\delta\left(c-c_{0}\right)$ in (21) for some $c_{0}>0$, where $\delta(c)$ is the one-dimensional Dirac measure.

We shall prove the contraposition, namely that if we assume

$$
{ }^{v} c_{0} ; m(c) \neq \delta\left(c-c_{0}\right),
$$

then $\mu$ is not $L$-ergodic.

From the assumption, there exists $c^{\prime}>0$ such that $m\left(\left(0, c^{\prime}\right]\right)>0$ and $m\left(\left(c^{\prime}, \infty\right)\right)>0$. Using this $c^{\prime}$, we put; 


$$
\mu^{\prime}(A)=\int_{\left(0, c^{\prime}\right\}} \mu_{c}(A) d m(c)
$$

Evidently $\mu^{\prime} \leqslant \mu$. Moreover $\mu^{\prime} \neq 0$, because $\mu^{\prime}\left(L^{*}\right)=m\left(\left(0, c^{\prime}\right]\right)>0$. Thus, in order to prove that $\mu$ is not $L$-ergodic, we should only show $\mu \$ \mu^{\prime}$. For this purpose, we use the function $f(x)$ which we have defined in Prop. 20.1. As seen there, we have $\mu_{c}\left(f^{-1}(c)\right)=1$ (for ${ }^{{ }} c>0$ ).

Now, put $A=f^{-1}\left(\left(c^{\prime}, \infty\right)\right)$, then

$$
\mu_{c}(A)=\left\{\begin{array}{lll}
1 & \text { if } & c>c^{\prime} \\
0 & \text { if } & c \leqq c^{\prime}
\end{array}\right.
$$

Hence,

$$
\mu(A)=\int_{(0, \infty)} \mu_{c}(A) d m(c)=m\left(\left(c^{\prime}, \infty\right)\right)>0,
$$

while

$$
\mu^{\prime}(A)=\int_{\left(0, c^{\prime}\right\}} \mu_{c}(A) d m(c)=0 .
$$

This shows us that $\mu \$ \mu^{\prime}$.

c) is proved in a similar way. (q.e.d.)

Corollary. For O-invariant measures (except the Dirac measure), $L$-ergodicity is equivalent with $O$-ergodicity.

As we have shown here, two fundamental questions about ergodic decomposition of measures are solved for $O$-invariant measures (c.f. the beginning of $\S 21$ ).

However, for $L$-ergodic decomposition of $L$-quasi-invariant measures, we have no solution up to the present time.

Even for the rotation of $L$, if we consider $O$-quasi-invariant measures (instead of $O$-invariant measures), we do not know how to solve these questions.

\section{REFERENCES}

[1] Segal, I. E., “Tensor algebras over Hilbert spaces. I." Trans. Amer. Math. Soc. 81 (1956) pp. 106 134.

[2] - "Distributions in Hilbert space and canonical systems of operators." Trans. Amer. Math. Soc. 88 (1958) pp. 12 41.

[3] Fukutome, H., "On the representation of the canonical commutation relation of Bose fields." Progress of Theoretical Physics, 23-6 (1960) pp. 989 1002.

[4] Hopf, E., "Ergodentheorie." Springer. Berlin (1937). 
[5] Neumann, J. von. "Zur Operatorenmethode in der klassischen Mechanik." Ann. Math. 33 (1932) pp. 587 642. (J. von Neumann collected works vol. 2 Pergamon, London (1961) pp. 307 362).

[6] Sudakov, V. N., "Linear sets with quasi-invariant measure." (in Russian). Doklady Akademii Nauk 127 (1959) pp. 524 525.

[7] Weil. A., "L'integration dans les groupes topologiques et ses applications." Appendice I. Paris, Hermann (1951).

[8] Grothendieck, A., 'Produits tensoriels topologiques et espaces nucléaires'.' Mem. Amer. Math. Soc. 16 (1955) pp. 1 140.

[9] Mityagin, B. S., "Approximate dimension and bases in nuclear spaces." Russian Math. Surveys. XVI 4 (1961) pp. 59 128.

[10] Minlos, R. A., "Generalized random processes and their extension to measures." (in Russian) Trudy Moskov. Mat. Obšč 8 (1959) pp. 497 518

[11] Bourbaki, N., "Espaces vectoriels topologiques" II. Paris, Hermann (1955).

[12] Matsuura, S., “On weak measurability in functional spaces'.' Journal of Mathematics of Kyoto University. vol. 1 , No. 2 (1962) pp. 203 224.

[13] Rosanov, Yu. A., "On the density of one Gaussian measure with respect to another." Theory of Prob. and Appl. VIII $\mathbb{1}$ (1962) pp. 62 86.

[14] Feldman, J., "Equivalance and perpendicularity of Gaussian processes." Pacific Journal of Mathematics 8 (1958) pp. 699 708.

[15] Dudley, R. M., "Singular translates of measures on linear spaces." Zeitschrift für Wahrscheinlichkeitstheorie 3 (1964) pp. 128 137.

[16] Sudakov, V. N., "On a class of compacts in Hilbert space." (in Russian). Uspekhi Mat. Nauk 18 (1963) pp. 181 187.

[17] "On the characterization of quasi-invariance of measures in Hilbert space." (in Russian) ibid. pp. 188 190. 
